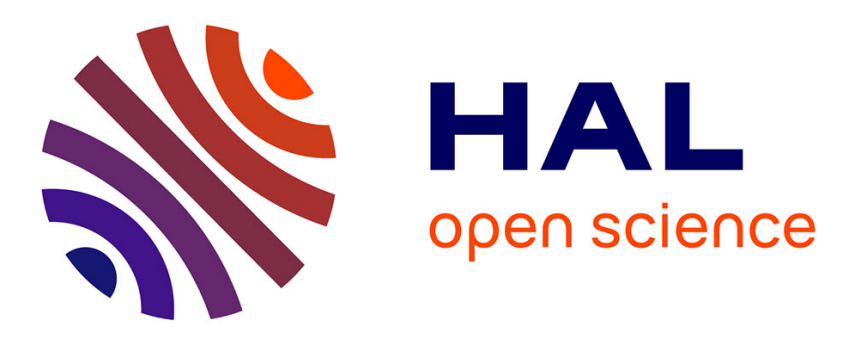

\title{
On the use of voice descriptors for glottal source shape parameter estimation
}

\author{
Stefan Huber, Axel Röbel
}

\section{To cite this version:}

Stefan Huber, Axel Röbel. On the use of voice descriptors for glottal source shape parameter estimation. Computer Speech and Language, In press, 0885-2308, www.sciencedirect.com/science/article/pii/S0885230813000776. 10.1016/j.csl.2013.09.006 . hal00865343

\section{HAL Id: hal-00865343 \\ https://hal.science/hal-00865343}

Submitted on 21 May 2019

HAL is a multi-disciplinary open access archive for the deposit and dissemination of scientific research documents, whether they are published or not. The documents may come from teaching and research institutions in France or abroad, or from public or private research centers.
L'archive ouverte pluridisciplinaire HAL, est destinée au dépôt et à la diffusion de documents scientifiques de niveau recherche, publiés ou non, émanant des établissements d'enseignement et de recherche français ou étrangers, des laboratoires publics ou privés. 


\title{
On the use of voice descriptors for glottal source shape parameter estimation ${ }^{\text {is }}$
}

\author{
Stefan Huber*, Axel Roebel ${ }^{* *}$ \\ Sound Analysis/Synthesis Team, IRCAM-CNRS-UPMC STMS, 1, place Igor Stravinsky, 75004 Paris, France
}

Received 24 April 2013; received in revised form 1 August 2013; accepted 18 September 2013

\begin{abstract}
This paper summarizes the results of our investigations into estimating the shape of the glottal excitation source from speech signals. We employ the Liljencrants-Fant (LF) model describing the glottal flow and its derivative. The one-dimensional glottal source shape parameter $R_{d}$ describes the transition in voice quality from a tense to a breathy voice. The parameter $R_{d}$ has been derived from a statistical regression of the $\mathrm{R}$ waveshape parameters which parameterize the LF model. First, we introduce a variant of our recently proposed adaptation and range extension of the $R_{d}$ parameter regression. Secondly, we discuss in detail the aspects of estimating the glottal source shape parameter $R_{d}$ using the phase minimization paradigm. Based on the analysis of a large number of speech signals we describe the major conditions that are likely to result in erroneous $R_{d}$ estimates. Based on these findings we investigate into means to increase the robustness of the $R_{d}$ parameter estimation. We use Viterbi smoothing to suppress unnatural jumps of the estimated $R_{d}$ parameter contours within short time segments. Additionally, we propose to steer the Viterbi algorithm by exploiting the covariation of other voice descriptors to improve Viterbi smoothing. The novel Viterbi steering is based on a Gaussian Mixture Model (GMM) that represents the joint density of the voice descriptors and the Open Quotient $(O Q)$ estimated from corresponding electroglottographic (EGG) signals. A conversion function derived from the mixture model predicts $O Q$ from the voice descriptors. Converted to $R_{d}$ it defines an additional prior probability to adapt the partial probabilities of the Viterbi algorithm accordingly. Finally, we evaluate the performances of the phase minimization based methods using both variants to adapt and extent the $R_{d}$ regression on one synthetic test set as well as in combination with Viterbi smoothing and each variant of the novel Viterbi steering on one test set of natural speech. The experimental findings exhibit improvements for both Viterbi approaches.
\end{abstract}

(C) 2013 Elsevier Ltd. All rights reserved.

Keywords: Glottal source; Voice quality; $R_{d}$ shape parameter; LF model; Viterbi smoothing

\section{Introduction}

The production of voiced human speech can be approximately modelled by assuming the glottal source as excitation and the vocal tract as filtering element. The convolution of one glottal flow cycle with an impulse train produces the glottal source waveform. The speech production model is completed by the convolution of the glottal excitation source with the impulse responses of the vocal-tract filter (VTF) as well as the radiation filters at lips and nostrils level. To

\footnotetext{
This paper has been recommended for acceptance by RKM.

* Corresponding author. Tel.: +33 (0)1 4478 4966; fax: +33 (0)1 44781540.

** Principal corresponding author. Tel.: +33 (0) 144784845 .

E-mail addresses: stefan.huber@ircam.fr (S. Huber), axel.roebel@ircam.fr (A. Roebel).
} 
solve the problem of estimating all components that are used in this model from a recorded speech signal a multitude of approaches have been proposed. However, for the moment none of these algorithms is sufficiently robust to allow for a reliable analysis of natural speech. In the following we will address a number of problems and investigate into means to obtain a physiologically consistent estimate of a glottal pulse shape parameter from synthetic and natural speech signals.

Voice qualities with a tense/pressed, modal/normal or relaxed/breathy phonation type are distinguishable by different shapes of the deterministic part of the glottal excitation source. Glottal source shapes can be efficiently described by one-dimensional parameterization techniques like the Normalized Amplitude Quotient (NAQ) (Alku et al., 2002) or the $R_{d}$ parameter (Fant et al., 1994; Fant, 1995). We use the latter to parameterize the LF model (Fant et al., 1985) describing the glottal volume-velocity flow and its derivative.

In our experiments with natural speech signals we observed the importance to cover extreme adducted and abducted phonations. This requires to extend the normal $R_{d}$ range [0.3, 2.7] (Fant, 1995) to lower $R_{d}$ values up to $R_{d}=0.1$ (extremely tense adducted phonation) and to higher $R_{d}$ values up to $R_{d}=6.0$ (extremely relaxed abducted phonation). The upper $R_{d}$ range (Fant et al., 1994) for $R_{d}>2.7$ is required to describe abducted phonations occuring mainly at phoneme transitions as well as at word and speaking pause boundaries. The $R_{d}$ range extension covers more glottal source shapes contained in the analyzed speech signal and augments thus the robustness of the $R_{d}$ estimation.

Unfortunately, the equations defining the $R_{d}$ regression (Fant et al., 1994; Fant, 1995) do not produce smooth contours of the $\mathrm{R}$ waveshape parameters when changing $R_{d}$ continuously between the normal and the upper $R_{d}$ range. To coherently cover an extended $R_{d}$ range $[0.1,6.0]$ we use our equations recently proposed in Huber et al. (2012). In this study we discuss more in detail the range adaptation of the $R_{d}$ parameter regression. Additionally, we introduce a second variant of the adapted and extended $R_{d}$ regression.

Moreover, to avoid local instabilities of the $R_{d}$ estimator we propose to apply Viterbi smoothing (Forney, 1973) since even with the extended $R_{d}$ range the results are often perturbed by implausible jumps. However, each of our $R_{d}$ estimation methods can, under some conditions, be systematically skewed. In addition, Viterbi smoothing cannot correct possibly skewed or biased $R_{d}$ contours in longer time segments. Therefore we propose to steer the Viterbi algorithm by exploiting the covariation of other voice descriptors. The latter are used to train GMMs from which a second $R_{d}$ estimate is predicted. This defines an additional prior $R_{d}$ probability used to steer Viterbi smoothing.

To estimate $R_{d}$ we use the phase minimization based paradigm established in Degottex et al. (2010), Degottex (2010) and Degottex et al. (2011). It is based on minimizing the mean squared phase errors present in the spectrum when matching synthesized glottal formants against a strictly harmonic representation of voiced speech. The $R_{d}$ value that is used to synthesize the glottal formant resulting in the lowest remaining phase error is selected as estimated $R_{d}$ value per frame. The baseline method called MSPD2I1 in Huber et al. (2012) and three recently in Huber et al. (2012) proposed phase minimization variants called MSPD2I0, MSPD2I2 and MSPD2IX are examined together with the two $R_{d}$ regression variants on an objective evaluation test set of synthetic speech signals.

Additionally, we examine the performance of Viterbi smoothing and the novel Viterbi steering using the four phase minimization methods to estimate $R_{d}$ on natural speech. The evaluation is similar to Fröhlich et al. (2001), Ó Cinnéide (2012) and Kane and Gobl (2013a). It is based on comparing the $O Q$ estimates derived from the $R_{d}$ curves $\left(O Q_{R_{d}}\right)$ with the $O Q$ estimates derived from an analysis of synchronously to the audio waveforms recorded EGG signals $\left(O Q_{E G G}\right)$. The objective of this study is to determine the best performing parameterization for Viterbi smoothing and the novel Viterbi steering as well as the most robust phase minimization method.

The article is organized as follows. The glottal source shape parameter $R_{d}$ and the LF model parameterization are detailed in Section 3. The basic model for the human speech production is introduced in Section 3. The phase minimization based methods to estimate the $R_{d}$ parameter are explained in Section 4 . The adaptation and the range extension of the $R_{d}$ parameter regression are discussed in Section 5. The optimized $R_{d}$ estimation using Viterbi smoothing and the novel Viterbi steering is explained in Section 6. The evaluation results are presented in Section 7 and 8 . The summary and the conclusions about this work are given in Section 9.

\section{LF model parameterization}

The R waveshape parameters $R_{a}, R_{k}$ and $R_{g}$ parameterize the LF model (Fant et al., 1985, 1994; Fant, 1995). $R_{a}$ is a relative measure of the return phase duration which is correlated to the spectral tilt of natural human speech. $R_{k}$ is 
defined by the ratio of the decay time to the rise time of the glottal pulse and describes its asymmetry. $R_{g}$ is an inverse measure of the glottal pulse rise time (Fant et al., 2000).

The parameterization of $R_{d}$ is determined by means of a statistical regression on values of the $\mathrm{R}$ waveshape parameter set (Fant and Liljencrants, 1994; Fant, 1995) that were observed for voiced natural speech (Gobl, 1988; Karlsson, 1990). It is based on exploiting systematic covariations of the R parameters present in the studied speech corpora. Fant introduced in Fant and Liljencrants (1994) and Fant (1995) equations to compute approximate R parameter values from an $R_{d}$ value, denominated with the subscript $p$ as 'predicted' $\mathrm{R}$ waveshape parameters $R_{a p}, R_{k p}$, and $R_{g p}$. We employ the term $R_{*} p$ waveshape parameter set. The equations how to calculate the parameters $R_{a p}$ and $R_{k p}$ from an estimated $R_{d}$ value for the normal $R_{d}$ range [0.3, 2.7] are given in Fant (1995). A definition following the explanations given in Fant (1995) how to compute $R_{g p}$ for the normal $R_{d}$ range can be found in Gobl (2003) and Degottex (2010). The R waveshape parameter set for the upper $R_{d}$ range ]2.7, 5] was proposed by Fant in Fant et al. (1994).

Fant proposed in Fant (1997) two possibilities to define the open quotient from the R waveshape parameter set. The reduced form $O Q_{i}$ defines the open quotient

$$
O Q_{i}=\frac{t_{e}}{T_{0}}=\frac{\left(1+R_{k p}\right)}{2} \cdot R_{g p}
$$

at the time instant of the maximum negative excitation $t_{e}$ of the glottal flow derivative, normalized by the fundamental period $T_{0}$. The complete form $O Q_{e}$ takes additionally into account the time of the return phrase $t_{a}$ to define the open quotient

$$
O Q_{e}=\frac{\left(t_{e}+t_{a}\right)}{T_{0}}=\frac{\left(1+R_{k p}\right)}{2} \cdot R_{g p}+R_{a p}
$$

The $R_{d}$ parameter is highly correlated with the time instant $t_{e}$ corresponding to $O Q_{i}$. Of high perceptual importance is the ratio of the peak $U_{0}$ of the glottal volume-velocity flow and the negative peak $E_{e}$ of the glottal flow derivative (Fant et al., 1994). It can be interpreted as effective pulse declination time $T_{d}=U_{o} / E_{e}$ by projecting the instants of both peaks in time to the time axis (Fant, 1997). The $R_{d}$ parameter can be expressed as $F_{0}$-normalized glottal waveshape parameter (Fant, 1995)

$$
R_{d}=\frac{U_{0}}{E_{e}} \cdot \frac{F_{0}}{110}=\left(\frac{1}{0.11}\right) \cdot\left(\frac{T_{d}}{T_{0}}\right)
$$

The scaling factor $1 / 110$ corresponds to $F_{0}=110 \mathrm{~Hz}$ as a typical average in male speech (Gobl, 1988; Fant, 1997). The direct ratio in amplitude measure relates $U_{0}$ and $E_{e}$ to the $T_{d}$-related Amplitude Quotient $(A Q)$ in Alku and Vilkman (1996) and the $R_{d}$-related Normalized Amplitude Quotient (NAQ) in Alku et al. (2002).

\section{Human voice production model}

The deterministic part of the voice production model used for the analysis consists of an extended source-filter model for stationary speech in the spectral domain

$$
S(\omega)=G_{R d}(\omega) \cdot C(\omega) \cdot L(\omega) \cdot H\left(\omega, F_{0}, D\right),
$$

with $\omega$ being the angular frequency. Eq. (4) defines the deterministic part of the voice production model which is composed of a representation of the following components. The shape parameter $R_{d}$ (Fant, 1995) parameterizes the Liljencrants-Fant (LF) (Fant et al., 1985) pulse model of the glottal flow $\left(G_{R d}(\omega)\right.$ ). The vocal tract transfer function is denoted as $C(\omega)$. It is assumed to have a minimum phase filter response. The radiation at lips and nostrils level is given by an approximate representation being $L(\omega)=j \omega$. The harmonic structure parameterized with the fundamental frequency $F_{0}$ and the delay between pulse sequence and frame center in terms of the phase delay $D$ of the fundamental sinusoid is represented by $H\left(\omega, F_{0}, D\right)$. 


\section{Glottal pulse parameter estimation}

The $R_{d}$ parameter estimation algorithms based on the objective functions for phase minimization that will be used in this study have been completely described in Degottex (2010), Degottex et al. (2011) and Huber et al. (2012). In this section we will only explain the basic scheme required for the following investigations presented in this paper.

The algorithm constructs a sinusoidal model of a speech signal frame. It is transformed into a harmonic model describing a single pitch period with a sampling rate that falls onto a harmonic grid at position $2 K+2$. The harmonic model is assumed to be noise free for each harmonic $\mathrm{k}$ up to $K$. In the experiments presented in this study we determine the highest harmonic sinusoidal partial $K$ by rounding the ratio $8000 \mathrm{~Hz}$ to $F_{0}$ to the nearest integer value. According to Eq. (4) the voice production model can then be simplified into

$$
S(k)=G_{R d}(k) \cdot C(k) \cdot L(k) \cdot e^{j k D} \quad k \in[0,1, \ldots, K] .
$$

We use the procedure described in Stylianou (2001) to construct $S(k)$ from a signal frame by means of finding the parameter set having minimum error. The fundamental frequency $F_{0}$ is estimated using the approach presented in Yeh and Roebel (2004). The algorithm proceeds by means of testing the minimum phase property of the VTF spectrum that is obtained for a sufficiently compact grid of $R_{d}$ values:

$$
\hat{C}_{R d}(k)=\frac{S_{k}}{G_{R d}(k) \cdot j k} .
$$

The residual will represent for the correct $R_{d}$ parameter the minimum phase transfer function of the vocal tract filter (Degottex et al., 2011). The accurate value of $R_{d}$ is estimated on the convolutive residual which is described in detail in Degottex et al. (2011) and Huber et al. (2012). The objective function of each phase minimization algorithm estimates $R_{d}$ by means of minimizing the deviation of the convolutive residual from a minimum phase transfer function An additional constant factor is introduced as error by the simplification of $L(k)$ into $j k$ which does not affect the results. Please note that if the duration of the impulse response of the VTF is close to or above the period the evaluation of the minimum phase property of the VTF becomes problematic. Ambiguous solutions may arise in these cases which may lead to erroneous $R_{d}$ (contour) estimates. Therefore, higher fundamental frequencies $F_{0}$ decrease as reported in Huber et al. (2012) the robustness and the accuracy of the $R_{d}$ estimation.

An arbitrary delay $D$ is introduced into the voice production model of Eqs. (4) and (5). It depends on the delay between the pulse position and the frame center in terms of the phase delay D of the fundamental (Huber et al., 2012). A 2nd order difference operator compensates the dependency on the pulse position but introduces a high-pass filter. Thus, subsequent integrations are required to suppress the influence of the high-pass filter.

The denomination Mean Squared Phase Differentiation Integration specifies the acronym MSPDI. The number of differentiation or integration steps are indicated by each subsequent number after D and respectively I. The application of first 2 differentiations and then 0,1 , and respectively 2 integration steps on the phase errors of the convolutive residual is contained in the acronyms of the three phase minimization methods MSPD2I0, MSPD2I1, and MSPD2I2 (Huber et al., 2012). The different error measures of this three method are combined by the method MSPD2IX in form of a weighted sum.

\section{Adaptation variants of the extended range $R_{d}$ regression}

Fig. 2 of Fant (1995) depicts the contour of the $\mathrm{R}$ waveshape parameters and for $O Q$ for the $R_{d}$ range [0.3, 5], using only 10 sampling points. It is the sole figure we could find in the literature illustrating these contours established by Fant for both the normal and the upper $R_{d}$ range. However, joining the curves for the normal and the upper $R_{d}$ range of the parameters $R_{k p}, R_{g p}$ and $O Q$ reveals a discontinuity at the interconnection point $R_{d}=2.7$, shown in Fig. 1 . The parameter contour of $O Q$ for the upper $R_{d}$ range (Fant et al., 1994) does not fit to $O Q$ for the normal $R_{d}$ range (Fant, 1995), neither to $O Q_{e}$ in complete form nor to $O Q_{i}$ in reduced form (Fant, 1997).

In Huber et al. (2012) we proposed an adaptation of the equations defining the computation of the $R * p$ waveshape parameter set to establish continuous parameter curves when changing $R_{d}$ between both ranges. Additionally, we extended the $R_{d}$ range to [0.01,6] to cover more extreme tense or breathy voice qualities. This requires to set $R_{a p}$ for $R_{d}<0.21$ to zero to avoid a negative return phase $t_{a}$. We adapt the $R_{g p}$ curves of the normal and the upper $R_{d}$ range at the minimum of the convex function of $R_{g p}$ for the normal $R_{d}$ range at $R_{d}=1.8476$. In Huber et al. (2012) we rounded 


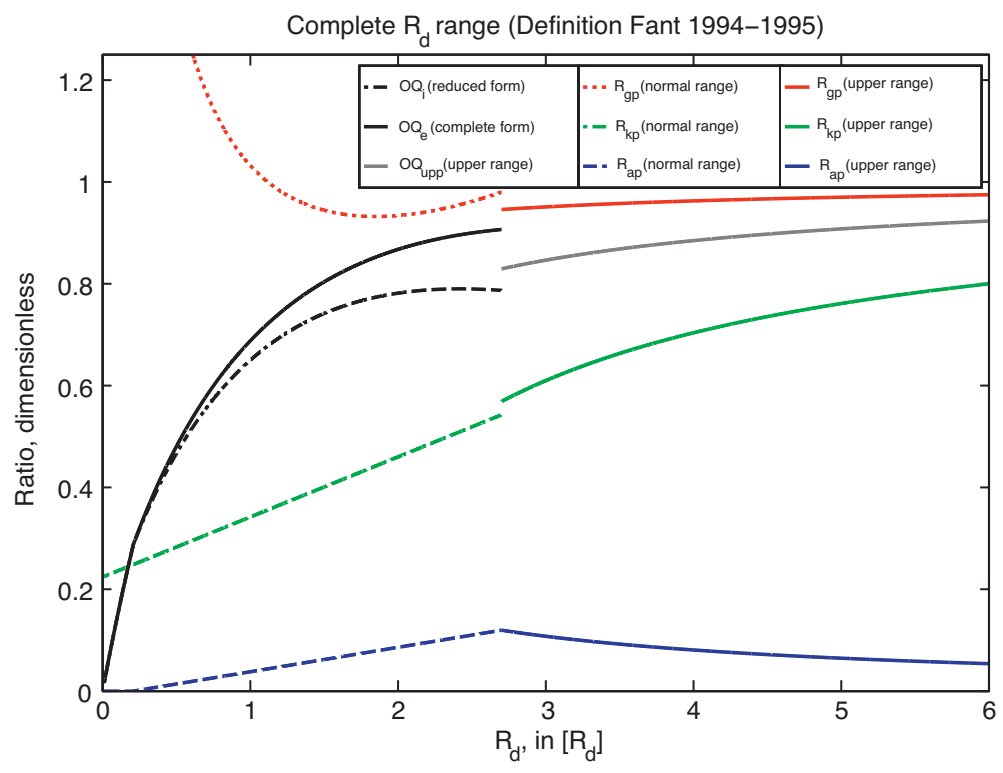

Fig. 1. Original waveshape $R_{*} p$ parameter contours.

this adaptation point to $R_{d}=1.85$. An offset of $9.3552 \times 10^{-3}$ has to be added to the $R_{g p}$ contour of the upper $R_{d}$ range to compensate for a remaining difference. Eqs. (7), (8), (9), and (10) follow our adaptation proposed in Huber et al. (2012), denoted here as variant 1 . It requires to add an offset of $-4.2753 \times 10^{-2}$ to $R_{k p_{2.70}}$ of the upper $R_{d}$ range.

In this paper we introduce adaptation variant 2 which takes into account that according to Fant et al. (1994) $R_{k p}$ for the upper $R_{d}$ range depends on $R_{g p}$. We adapt $R_{k p}$ to depend on the upper range equation not at $R_{d}=2.7$ as defined in Fant et al. (1994) but already at $R_{d}=1.8476$ to be more conform with our introduced $R_{d}$ regression adaptation of $R_{g p}$ in Huber et al. (2012). $R_{k p}$ for adaptation variant 2 is consequently denominated as $R_{k p_{1.85}}$. An offset compensation of $+4.2753 \times 10^{-2}$ adapts $R_{k p_{1.85}}$ at the upper $R_{d}$ range accordingly. The contours of our proposed $R * p$ parameter adaptation for both variants are shown in Fig. 2.

Please note that the $O Q$ contour of the reduced form $O Q_{i}$ derived from the original $R_{d}$ regression of Fant $(1995,1997)$ exhibits for the normal $R_{d}$ range a maximum of $O Q_{i}=0.790$ already at $R_{d}=2.42$ and a lower value of $O Q_{i}=0.787$ at $R_{d}=2.70$. But, $O Q$ should increase over the $R_{d}$ range from lower values for tense adducted to higher values for breathy abducted phonations (Henrich et al., 1999; Doval et al., 2006). The decrease of $O Q_{i}$ for the $R_{d}$ range [2.42, 2.70] introduces ambiguities into pulse parameter estimation algorithms. Our $R_{d}$ regression adaptation variants suppress these ambiguities by establishing a strictly increasing $O Q_{i}$ contour over the whole $R_{d}$ range. We find for the reduced $O Q_{i}$ form defined in Eq. (1) maximum values of $O Q_{i}=0.90$ at $R_{d}=6.0$ for variant 1 and $O Q_{i}=0.95$ for variant 2.

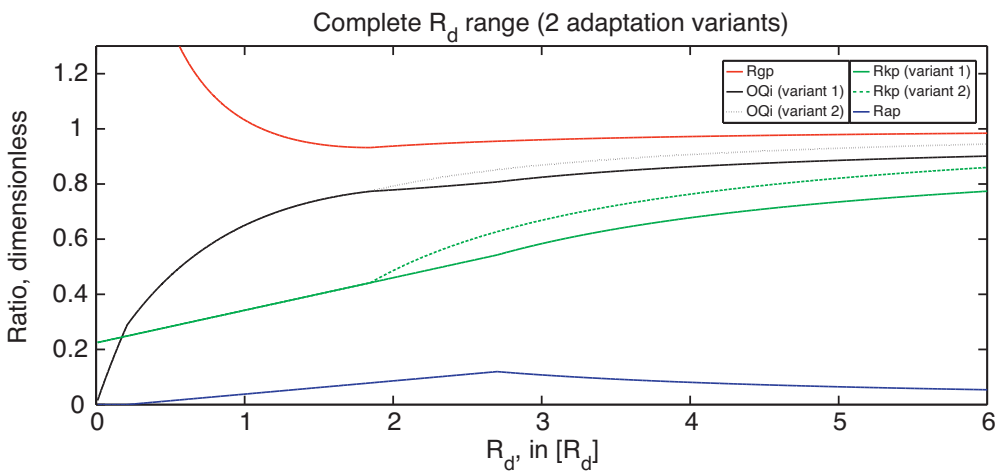

Fig. 2. Adapted waveshape $R_{*}^{*}$ parameter contours. 
Our proposed set of equations with a more exact listing of the offset and border values as reported in Huber et al. (2012) to define the adaptation of the $R_{d}$ waveshape parameter regression for the extended $R_{d}$ range and both adaptation variants is:

$$
\begin{aligned}
& R_{a p}= \begin{cases}0 & \forall 0.01 \leq R_{d}<0.21 \\
\frac{\left(-1+4.8 \cdot R_{d}\right)}{100} & \forall 0.21 \leq R_{d} \leq 2.70 \\
\frac{\left(32.3 / R_{d}\right)}{100} & \forall 2.70<R_{d} \leq 6.00\end{cases} \\
& O Q_{u p p}=1-\frac{1}{\left(2.17 \cdot R_{d}\right)} \quad \forall 1.8476 \leq R_{d} \leq 6.00
\end{aligned}
$$

Variant 1:

$$
\begin{aligned}
& R_{k p_{2.70}}= \begin{cases}\frac{\left(22.4+11.8 \cdot R_{d}\right)}{100} & \forall 0.01 \leq R_{d} \leq 2.70 \\
\left(2 \cdot R_{g p} \cdot O Q_{u p p}\right)-1.0428 & \forall 2.70<R_{d} \leq 6.00\end{cases} \\
& R_{g p}= \begin{cases}\frac{0.25 \cdot R_{k p_{2.70}}}{\left((0.11 \cdot R d) /\left(0.5+1.2 \cdot R_{\left.\left.k p_{2.70}\right)\right)-R_{a p}}\right.\right.} & \forall 0.01 \leq R_{d} \leq 1.8476 \\
9.3552 \times 10^{-3}+\frac{596 \times 10^{-2}}{7.96-2 \cdot O Q_{u p p}} & \forall 1.8476<R_{d} \leq 6.00\end{cases}
\end{aligned}
$$

Variant 2:

$$
\begin{aligned}
& R_{k p_{1.85}}= \begin{cases}\frac{\left(22.4+11.8 \cdot R_{d}\right)}{100} & \forall 0.01 \leq R_{d} \leq 1.8476 \\
\left(2 \cdot R_{g p} \cdot O Q_{u p p}\right)-0.9572 & \forall 1.8476 \leq R_{d} \leq 6.00\end{cases} \\
& R_{g p}= \begin{cases}\frac{0.25 \cdot R_{k p_{1.85}}}{\left((0.11 \cdot R d) /\left(0.5+1.2 \cdot R_{k p_{1.85}}\right)\right)-R_{a p}} & \forall 0.01 \leq R_{d} \leq 1.8476 \\
9.3552 \times 10^{-3}+\frac{596 \times 10^{-2}}{7.96-2 \cdot O Q_{u p p}} & \forall 1.8476<R_{d} \leq 6.00\end{cases}
\end{aligned}
$$

\section{Viterbi smoothing and steering}

\subsection{Viterbi smoothing}

In this paper we denominate the well accepted Viterbi algorithm (Forney, 1973) as standard Viterbi smoothing. We distinct it from our novel approach to steer the Viterbi algorithm which we denominate as Viterbi steering accordingly. Viterbi steering exploits the covariation of voice descriptors and voice quality features and will be introduced in Section 6.2.

The $R_{d}$ parameter estimation operates frame-based by selecting at each analysis step the glottal pulse shape corresponding to the lowest remaining residual phase error. Errors may arise from:

- Environmental or aspiration noise.

- General ambiguities from non-linear phase distortions present in the phase residual (Walker and Murphy, 2007; Ó Cinnéide et al., 2011).

- Situations where the $R_{d}$ parameterization of the LF model restricts the synthesized and estimated glottal source shapes to an efficient subspace of the LF model parameter space which does not cover the true glottal source contained in the signal.

- The fact that the precise minimum phase impulse response of the vocal tract cannot be observed with the real cepstrum used by the phase minimization methods (Degottex, 2010) from signal parts where only few stable harmonic partials 
are available before being masked by noise. This situation occurs predominantly for higher fundamental frequencies, at phoneme transitions or at word and speaking pause boundaries. Moreover, the stationarity of the vocal-tract filter over the length of the analysis window may not be anymore valid at these situations. The phase minimization paradigm (Degottex et al., 2011) may systematically be mislead in such segments.

These random influences can partially be reduced by smoothing over time with the Viterbi algorithm, as long as these problems are present over a relatively short time segment. We define the probabilistic model of standard Viterbi smoothing as follows:

Observation probability $P(O \mid X)$

We approximate the speech production model using a grid of $R_{d}$ values. Each of the $N_{R_{d i}} R_{d}$ values represents a hidden state $X_{i}$ of a finite-state Markov process that defines the random process to establish the Viterbi algorithm. The phase error of the convolutive residual determines the log-likelihood of the observation. The probabilistic distribution of the observation is configured so that the minimum error of the residual phase $E_{R d}=0$ has maximum probability. The emitted observations over time span up the lattice over which the Viterbi algorithm determines the optimal path representing the lowest overall error.

Transition probability $P\left(X_{n} \mid X_{n-1}\right)$

The transition probability is described as a function of the $R_{d}$ parameter slope $\Delta R_{d} / \Delta_{n}$, with $\Delta_{n}$ representing the time difference between two analysis frames such that the transition probability can consistently handle different STFT analysis step sizes. The probabilistic distribution of the transition is modelled as Gaussian with zero mean and variance $\sigma_{T}^{2}$.

\section{Optimal Viterbi smoothing path}

The sequence of observations is segmented into regions of voiced speech. The voicing decision is based on the presence of frames containing valid glottal closure instants (GCI). We employ the SIGMA algorithm (Thomas and Naylor, 2009) to detect the GCIs. The evaluation of this work is restricted to only consider voiced segments having at least five consecutive voiced frames. The $R_{d}$ sequences having maximum probability are determined by applying the Viterbi algorithm independently to each voiced segment. The log-likelihood of each sequence is

$$
L(p)=\sum_{n} \log \left(P\left(O \mid X_{p}(n)\right) \cdot P\left(X_{p}(n) \mid X_{p}(n-1)\right)\right),
$$

where $n$ is the discrete time and $p$ is a path through the state space of the process. We insert into Eq. (13) the log probability function $E_{R d}$ of the observation probability $P(O \mid X)$ and scale its distribution with parameter $\alpha_{a}$. Respectively we include the probabilistic distribution $\Delta R_{d} / \Delta_{n}$ of the transition probability $P\left(X_{n} \mid X_{n-1}\right)$ with its scale parameter $\gamma_{g}$ to find

$$
\bar{L}(p)=-\sum_{n} \alpha_{a} \cdot E_{R_{d n}}+\gamma_{g} \cdot \frac{\Delta R_{d}}{\Delta_{n}}+C .
$$

The term $C$ is a constant gathering all the contributions of the constant scaling factors of the distributions. This constant term can be ignored by the Viterbi algorithm. We can factor out the scaling factor $\gamma_{g}$ of the log-likelihood of the transition which leaves the parameter $\alpha=\alpha_{a} / \gamma_{g}$ as control parameter. This defines the probability function that is used to perform Viterbi smoothing on all sequences $p$ :

$$
\overline{\bar{L}}(p)=-\sum_{n} \alpha \cdot E_{R_{d n}}+\frac{\Delta R_{d}}{\Delta_{n}} .
$$

The experimental setup of Section 8 and the test of Section 8.2.4 examines which value for $\alpha$ creates the best Viterbi smoothing results without the application of the novel Viterbi steering.

\subsection{Viterbi steering}

The novel steering of the Viterbi algorithm constitutes an extension of standard Viterbi smoothing presented in the preceding Section 6.1. Viterbi smoothing can augment the $R_{d}$ estimation robustness but it cannot correct a systematic bias present in longer time segments. We observe a possible systematic bias for each $R_{d}$ estimator predominantly in regions where only few stable harmonic sinusoids are available, e.g. at phoneme transitions, word and speaking 
pause boundaries or for higher fundamental frequencies. In this chapter we investigate into means to correct a possible systematic bias of the $R_{d}$ estimation in the mentioned problematic regions.

The phase minimization paradigm (Degottex et al., 2011) requires a precise estimation of the minimum phase response of the VTF from the observed partials. This condition may not be given if only few stable harmonic sinusoids are observable. Therefore we examined the covariation of additional voice descriptors in terms of a strong positive or negative correlation with a robust $O Q$ estimate. The latter defines partially the shape of the deterministic part of the glottal excitation source. We use the recordings of the speakers BDL, JMK and SLT of the CMU Arctic speech database (Kominek and Black, 2004) which provides simultaneously recorded speech waveforms and EGG signals. To estimate $O Q$ from the corresponding EGG signals we use the DECOM method (Henrich et al., 2004). We derive the $O Q_{E G G}$ contours from all available phrases of all three speakers for all voiced segments using the voicing decision described in Section 6.1.

As a proof-of-concept for the possibility to exploit specific speech signal features for the estimation of the $R_{d}$ parameter we establish a machine learning approach to aid Viterbi smoothing. By using a statistical model we exploit the information measured from additional voice descriptors that are correlated with $O Q_{E G G}$. The utilized features and the $O Q_{E G G}$ originate both from the same underlying glottal gestures which reflect the physiological mechanisms of human speech production at the larynx (Laver, 1980; Gobl and Chasaide, 1992). A function to predict a second $O Q$ estimate from the voice descriptor set is derived from the trained statistical model.

\subsubsection{Covariation voice descriptors}

From an extensive analysis of different voice descriptors and combinations in-between them on the CMU databases we determined several voice descriptors. Each selected feature demonstrated to be highly positively correlated with the $O Q_{E G G}$ reference, except the Voiced/Unvoiced Frequency $(V U F)$ boundary (Roebel, 2010; Stylianou, 2001) which shares a negative correlation. All proposed voice descriptors are not influenced by a lower number of stable harmonic sinusoids and are therefore well suited to exploit their covariation with the shape of the glottal excitation source.

H1-H2: The amplitude difference in $\mathrm{dB}$ of the first two harmonic partials $\mathrm{H} 1$ and $\mathrm{H} 2(\mathrm{H} 1-\mathrm{H} 2)$. According to Henrich et al. (2001) it is a reliable spectral correlate of $O Q$. H1-H2 proved to contribute to the discrimination between breathy and tense voice qualities in Scherer et al. (2012). We measure the relation directly in the magnitude spectrum and do not apply inverse filtering to measure $\mathrm{H} 1{ }^{*}-\mathrm{H} 2{ }^{*}$ from the corresponding glottal source signal as in Fant (1995), Hanson (1995) and Henrich et al. (2001). This avoids possible problems with inverse filtering (Rothenberg, 1972; Alku, 1992; Drugman et al., 2008), while the direct measure of H1 in the magnitude spectrum is according to Keating and Esposito (2006) influenced by the first formant F1. To smooth the direct H1-H2 measure we apply a median filter of order 5 .

3 MFCC bins: With the sum of the 3rd, 4th and 6th MFCC bin (Ellis, 2005) we seek to model the spectral slope (Scherer et al., 2012) or the spectral tilt (Murphy, 2001) to reflect the amplitude continuation of the spectral envelope which is correlated to a tense, modal or breathy phonation. However, neither a regression on the slope of the spectral peaks as in Scherer et al. (2012) nor the utilization of the spectral tilt measures $R_{14}$ or $R_{24}$ as in Murphy (2001) nor other related measurements were able to achieve an overall correlation to the $O Q_{E G G}$ reference being as high and robust as the summed combination of the 3rd, 4th and 6th MFCC bin (Ellis, 2005). Apparently, the proposed summation of the three MFCC bins is less influenced by the variation of the vocal tract formants.

$\mathbf{F}_{0}$ : The fundamental frequency $F_{0}$ shares according to Fant et al. (1994) systematic dependencies with $U_{0}$ and $E_{e}$, and hence with $R_{d} . U_{0}$ has a close relation to the amplitude of the voice fundamental while $E_{e}$ is the basic determinant of formant amplitudes. In Laver (1968) the laryngeal settings are categorized into phonation types, pitch ranges and loudness ranges. Therefore, the larynx as physiological foundation of the voice not just originates the different voice qualities but serves as well as a determinant of $F_{0}$ and sound pressure level (SPL) contours. Speakers favour a particular pitch range for each phonation type (Laver, 1968; Childers and Lee, 1991). However, different studies (Laver, 1980; Maddieson and Hess, 1987; Hanson et al., 1990) have shown that the relation between pitch and different phonation types is speaker-dependent. We estimate $F_{0}$ using the monophonic $F_{0}$ implementation based on the principles described in Yeh and Roebel (2004).

VUF: The Voiced/Unvoiced Frequency (VUF) boundary (Roebel, 2010; Stylianou, 2001) correlates with the voiced/unvoiced energy ratio and the bandwidth of the glottal formant. The $V U F$ is thus related to $E_{e}$ which determines the amount of generated sinusoidal energy (Fant, 1995), and the noise energy level which originates from turbulences created at the glottis, for example due to an imperfect glottal closure and a high airflow rate (Childers and Lee, 1991). 
A tense voice parameterized by low $R_{d}$ values originates a broad excitation spectrum with sinusoidal content present in higher frequency regions, while a relaxed voice parameterized by higher $R_{d}$ values originates only few harmonic sinusoidal partials in lower frequency regions (Fant, 1995). The VUF is estimated by a) detecting sinusoidal peaks (Zivanovic et al., 2004), b) measuring the sinusoid vs. noise energy ratio (SNE) in sub-bands of fixed and constant bandwidth, c) selecting as VUF the highest band for that the SNE is above a given threshold. The measured $V U F$ contour is smoothed using a median filter covering the time of half the length of the analysis window.

\subsection{2. $O Q_{G M M}$ prediction model}

The establishment of a formula that uses the proposed voice descriptor feature set to predict $R_{d}$ is very difficult. For example, the empiric formulation of Fant (1997) expresses the relation between the $H 1^{*}-H 2^{*}$ measure with $O Q$ by an exponential function. It implies a lower limit of $-5.73 \mathrm{~dB}$ for $\mathrm{H} 1^{*}-\mathrm{H} 2^{*}$ at $O Q=0.3$. However, from an informal examination on the CMU Arctic databases we measure $\mathrm{H} 1-\mathrm{H} 2$ and $O Q_{E G G}$ values below both limits. In Henrich et al. (2001) and Doval et al. (2006) it is shown that the relation between $\mathrm{H} 1^{*}-\mathrm{H} 2^{*}$ and $O Q$ is additionally influenced by the asymmetry coefficient $a_{m}$ representing the skewness of the glottal pulse. Moreover, recent studies (Kreiman et al., 2012, 2012b; Chen et al., 2013) suggest that the relationship is speaker-dependent, leading to positive and negative correlations, or situations where one parameter remains relatively constant while the other varies considerably.

We could not find an analytic formulation expressing the relation of $O Q$ with any of the other voice descriptors in the literature, and as well not for the complete set of the proposed voice descriptors. Thus, we employ a GMM to model the relation of the covariation feature set with the $O Q_{E G G}$ reference. A similar approach using Gaussian mixture modelling to predict glottal source signals has already been proposed in Thomas et al. (2009) and Gudnason et al. (2012). Per speaker we train one GMM on the covariation feature combination estimated on each voiced segment of the other two speaker databases and their corresponding $O Q_{E G G}$ estimates.

We distinct two feature sets:

Model 1 refers to the utilization of the voice descriptors $\mathrm{H} 1-\mathrm{H} 2, V U F$ and the sum of the 3rd, 4th and 6th MFCC bin.

Model 2 additionally includes the fundamental frequency $F_{0}$.

The GMM modelling is based on a modified version of the Voice Conversion system described in Lanchantin and Rodet $(2010,2011)$. We express the joint probability density $p\left(M, R \mid \lambda^{(Z)}\right)$ of the feature set $\mathrm{M}$ (either model 1 or 2 ) and the $O Q_{E G G}$ reference R, conditioned on the model parameters $\lambda$ with the weights $\alpha_{q}$, mean vector $\mu_{q}^{Z}$ and the covariance matrices $\Sigma_{q}^{Z}$ over $Q$ mixture sequences as

$$
p\left(M, R \mid \lambda^{(Z)}\right)=\sum_{q=1}^{Q} \alpha_{q} \cdot N\left(M, R ; \mu_{q}^{(Z)}, \Sigma_{q}^{(Z)}\right) .
$$

$Q$ is set to 6 mixture components for model 1 , and to respectively 8 mixture components for model 2 . The function

$$
F(m)=\sum_{q=1}^{Q} p_{q}^{m} m \cdot\left[\mu_{q}^{r}+\Sigma_{q}^{r m} \Sigma_{q}^{m m^{-1}}\left(m-\mu_{q}^{m}\right)\right]
$$

for the prediction of $O Q_{G M M}$ from a feature set is derived from the trained GMM model, with the conjoint data set of $\mathrm{M}$ and $\mathrm{R}$ being expressed by $Z=\left\{z_{k}\right\}, z_{k}=\left[m_{k}^{T} r_{k}^{T}\right]^{T}$.

\subsubsection{Viterbi steering implementation}

The prediction of $O Q_{G M M}$ values from each GMM model per speaker defines the additional prior probability $M_{\text {prior }}$ for the Viterbi algorithm. It is used to steer the standard Viterbi smoothing approach according to the prediction of $O Q_{G M M}$ using either the feature set for model 1 or model 2.

Prior $R_{d}$ probability $P(X \mid M)$

The $O Q_{G M M}$ prediction is transformed into the $R_{d}$ value range $\left(R_{d_{G M M}}\right)$. It is modelled as Gaussian with variance $\sigma_{P}^{2}$. It defines an additional prior $R_{d}$ probability from the $R_{d_{G M M}}$ prediction given the voice descriptor feature set $\mathrm{M}$ that is used to steer the Viterbi algorithm. A possibly occurring mean offset between the predicted $R_{d_{G M M}}$ and the estimated $R_{d}$ of each phase minimization method has to be compensated per voiced segment. The probabilistic modelling $M_{\text {prior }}$ 
is configured to have maximum probability if the value of $R_{d_{G M M}}$ and the $R_{d}$ value estimated by our phase minimization methods are congruent to each other. Differences between predicted and estimated $R_{d}$ lead to lower $M_{\text {prior }}$ probabilities.

\section{Optimal Viterbi steering path}

We insert the prior $R_{d}$ probability into Eq. (13) to find

$$
L(p)=\sum_{n} \log \left(P\left(O_{R_{d}} \mid X_{p}(n)\right) \cdot P\left(X_{p}(n) \mid M(n)\right) \cdot P\left(X_{p}(n) \mid X_{p}(n-1)\right)\right) .
$$

In the following we define the scale parameters of the log-likelihood function $L$ of the distribution. Parameter $\alpha_{a}$ represents the scale parameter of the error function $E_{R_{d}}$ of the observation probability $O_{R_{d}}$. The log-likelihood $M_{\text {prior }}$ of the prior $R_{d}$ probability is scaled by parameter $\beta_{b}$. Parameter $\gamma_{g}$ scales the distribution of the transition probability $P\left(X_{n} \mid X_{n-1}\right)$.

$$
\bar{L}(p)=-\sum_{n} \alpha_{a} \cdot E_{R_{d n}}+\beta_{b} \cdot M_{\text {prior }_{n}}+\gamma_{g} \cdot \frac{\Delta R_{d}}{\Delta_{n}}+C
$$

Again, the constant term $C$ can be ignored by the Viterbi algorithm. We factor out the scaling factor $\gamma_{g}$ to define the scaling factors $\alpha=\alpha_{a} / \gamma_{g}$ and $\beta=\beta_{b} / \gamma_{g}$ as control parameters of the Viterbi steering approach on all sequences $p$.

$$
\overline{\bar{L}}(p)=-\sum_{n} \alpha \cdot E_{R_{d n}}+\beta \cdot M_{\text {prior }_{n}}+\frac{\Delta R_{d}}{\Delta_{n}}
$$

The evaluation tests for the proposed novel Viterbi steering of Eq. (20) are presented and discussed in Section 8. We examine which values for $\alpha$ and $\beta$ result in the highest performance to estimate $R_{d}$ contours on natural speech using as evaluation data set the three CMU Arctic databases.

\section{Objective evaluation on a synthetic test set}

In this section we examine the performance of phase minimization algorithms to estimate $R_{d}$ on a synthetic test set similar to Degottex et al. (2011) and Huber et al. (2012). The four $R_{d}$ estimation methods MSPD2I0, MSPD2I1, MSPD2I2 and MSPD2IX (Huber et al., 2012) are evaluated with respect to their dependency on some characteristics present in speech signals. The influences of the fundamental frequency $F_{0}$, the number of observed stable harmonic sinusoids $N$, different configurations of the vocal tract filter, the glottal source noise $n^{\sigma_{g}}$ and the environmental noise $n^{\sigma_{e}}$ are investigated.

The estimation of glottal source characteristics depends as reported in Drugman et al. (2008) on the position of the glottal formant $F_{g}$ to the VTF formants, notably to the first formant $F_{1}$. To simulate the VTF influence we synthesize 16 synthetic vowels $C_{V T F}$ using Maeda's digital simulator (Maeda, 1982). Each $C_{V T F}$ is convolved with each glottal formant parameterized by an $R_{d}$ value within the range [0.1,6] on a grid of step size $R_{d}=0.1$. We synthesize at 10 different $F_{0}$ values within the range [80,293] Hz. 6 Gaussian noise levels $n$ between $[-50,-25] \mathrm{dB}$ are added to the voiced signal. Each noise level is applied to both noise influences $n^{\sigma_{s}}[n]$ and $n^{\sigma_{e}}[n]$.

The ratio of $V U F$ boundary to $F_{0}$ determines how many stable harmonic sinusoids $N$ are observable before being masked by noise. We evaluate the influence of $N$ for the range [3, 8] by restricting the $R_{d}$ estimation algorithm to observe $N$ partials. Additionally we simulate the position of the window with respect to the period in time on a grid of 4 different delays $\phi^{*}$ covering the range $[-0.5 \cdot T, 0.5 \cdot T]$. One synthetic test per $R_{d}$ regression variant and phase minimization method consists in total of $1,382,400$ single tests $\left(60 R_{d} \cdot 10 F_{0} \cdot 6 n^{\sigma_{g}}[n] / n^{\sigma_{e}}[n] \cdot 16 C_{V T F} \cdot 6 \mathrm{~N}\right.$. $4 \phi$ values). The results are presented in a compact manner by adding up the bias and standard deviation of the $R_{d}$ estimation errors as a function of the examined parameter.

\subsection{Examination on dependency in $F_{0}$}

Fig. 3 illustrates how higher fundamental frequencies $F_{0}$ lead to a constant increase in the error amount of wrongly estimated $R_{d}$ glottal source pulse shapes. The $R_{d}$ regression adaptation variant 2 (depicted for each method in dashdotted lines) performs in general worse than variant 1 (solid lines). The overall best performing methods over the complete frequency range of 80 to $293 \mathrm{~Hz}$ are MSPD2IX and MSPD2I2 under the utilization of adaptation variant 1. The method MSPD2IX using the less performant adaptation variant 2 even outperforms the baseline method MSPD2I1 


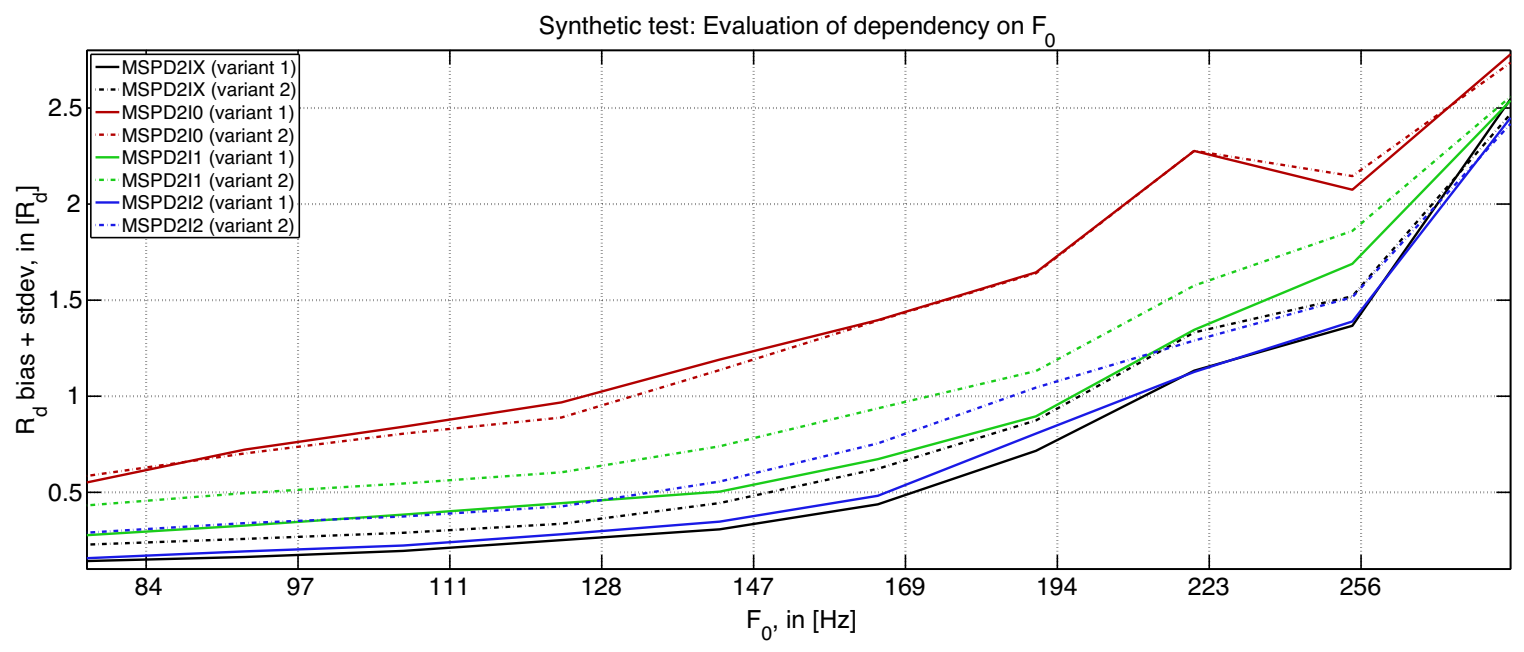

Fig. 3. Evaluation of $R_{d}$ estimation, $F_{0}$-dependency.

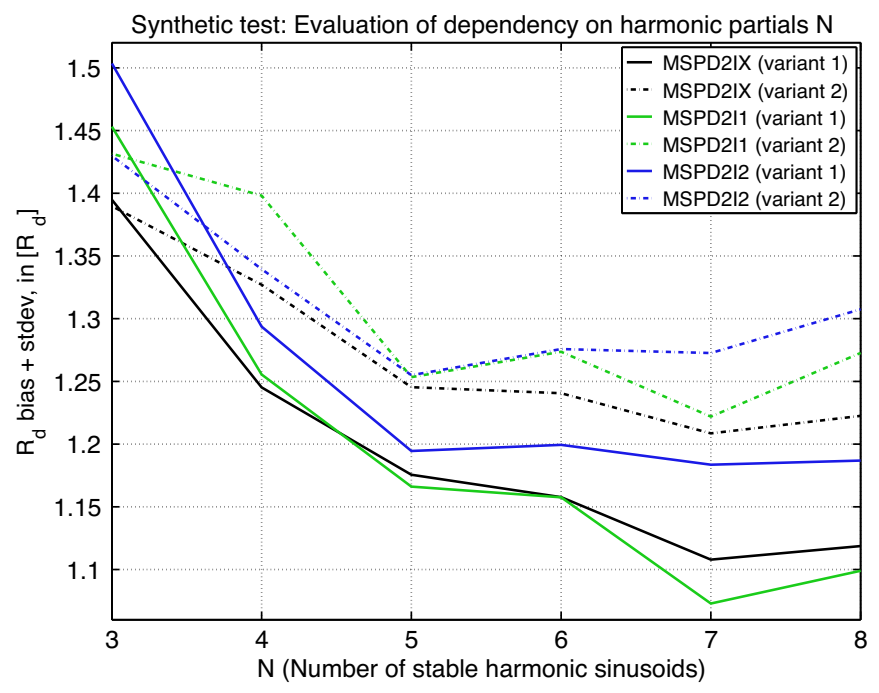

Fig. 4. Evaluation of $R_{d}$ estimation, N-dependency.

using adaptation variant 1 over the complete $F_{0}$ range. The latter yields overall similar results as MSPD2I2 using the less good performing adaptation variant 2. The method MSPD2I0 performs in general worst. The $R_{d}$ estimation results are relatively robust up to frequencies of $\sim 150-200 \mathrm{~Hz}$, above which more severe perturbations of the estimation accuracy are apparent (Drugman et al., 2008; Huber et al., 2012). If the duration of the impulse response of the VTF is close to or above the period the estimation of the VTF minimum phase property is less accurate. This leads to less robust estimations with increasing $F_{0}$.

\subsection{Examination on dependency in harmonic partials}

The same effect of a comparatively lower number of stable harmonic sinusoids $N$ and a less accurate estimation of the minimum phase property of the VTF is introduced with higher noise levels. We simulate these influences by varying $N$, shown in Fig. 4 . For the variation of $N$ depicted in Fig. 4 we set both the glottal source noise $n^{\sigma_{g}}$ and the environmental noise $n^{\sigma_{e}}$ to $n=1$. This corresponds to the lowest noise level of $-50 \mathrm{~dB}$ used in our synthetic test. With this low noise level the characteristics of natural speech are simulated but the misleading interference of noise is suppressed which is required to properly examine a different number of stable harmonic sinusoids $N$. 
Since MSPD2I0 demonstrated to perform worst it is omitted in Fig. 4 to provide a more clear presentation. The best performing methods MSPD2IX and MSPD2I1 indicate most obviously that a lower number of stable harmonic sinusoids $N$ leads to a higher amount of $R_{d}$ estimation errors. However, each method exhibits the lowest overall cumulated amount of $R_{d}$ estimation errors at $N=7$. This is justified by the fact that the harmonic sinusoids of $N \geq 8$ may already be covered by noise at level $-50 \mathrm{~dB}$. Again, the $R_{d}$ adaptation variant 2 leads to less good $R_{d}$ estimation results than adaptation variant 1 .

\subsection{Examination on dependency in voice quality}

Fig. 5 exemplifies how the objective function of each phase minimization based method is dependent on the phase differences of the LF model parameterized by different $R_{d}$ values over the complete $R_{d}$ range. A too high self-similarity of LF models parameterized by an R waveshape parameter set being close in value leaves the estimation method with little differentiation possibilities. Fig. 5 indicates that glottal source shapes in the $R_{d}$ range of $[0.3,2]$ are more dissimilar to each other than the glottal source shapes of the upper $R_{d}$ range of ]2.7,6] or tense phonations parameterized by our proposed $R_{d}$ range extension below the lower limit $R_{d}<0.3$ of the normal $R_{d}$ range.

This reflects to a certain extent the observations concluded with the $R_{d}$ confusion matrices of Huber et al. (2012): Broader error valleys of each objective function for phase minimization lead to a less robust $R_{d}$ estimation. The conceptual equivalent to the $R_{d}$ confusion matrices is shown by Fig. 7. The $R_{d}$ estimation error surfaces are spanned up frame-wise over time and reflect the behaviour of each objective function.

Finally we conclude that the overall less good $R_{d}$ estimation performance of $R_{d}$ adaptation variant 2 results from the fact that it renders higher $O Q$ values which proves for this synthetic test to suffer from a higher error rate. When examining Fig. 5 by visual inspection it is apparent that the phase minimization methods using adaptation variant 2 perform better for lower $R_{d}$ values in the normal $R_{d}$ range $[0.3,2.7]$ and gradually underperform more for higher $R_{d}$ values above $R_{d}>2.7$.

\section{Objective evaluation on natural speech}

In this section we evaluate the four phase minimization methods (Huber et al., 2012) on natural speech. Each method has estimated $R_{d}$ on each voiced segment of all available phrases of the CMU Arctic speech databases (Kominek and Black, 2004) BDL, JMK, and SLT.

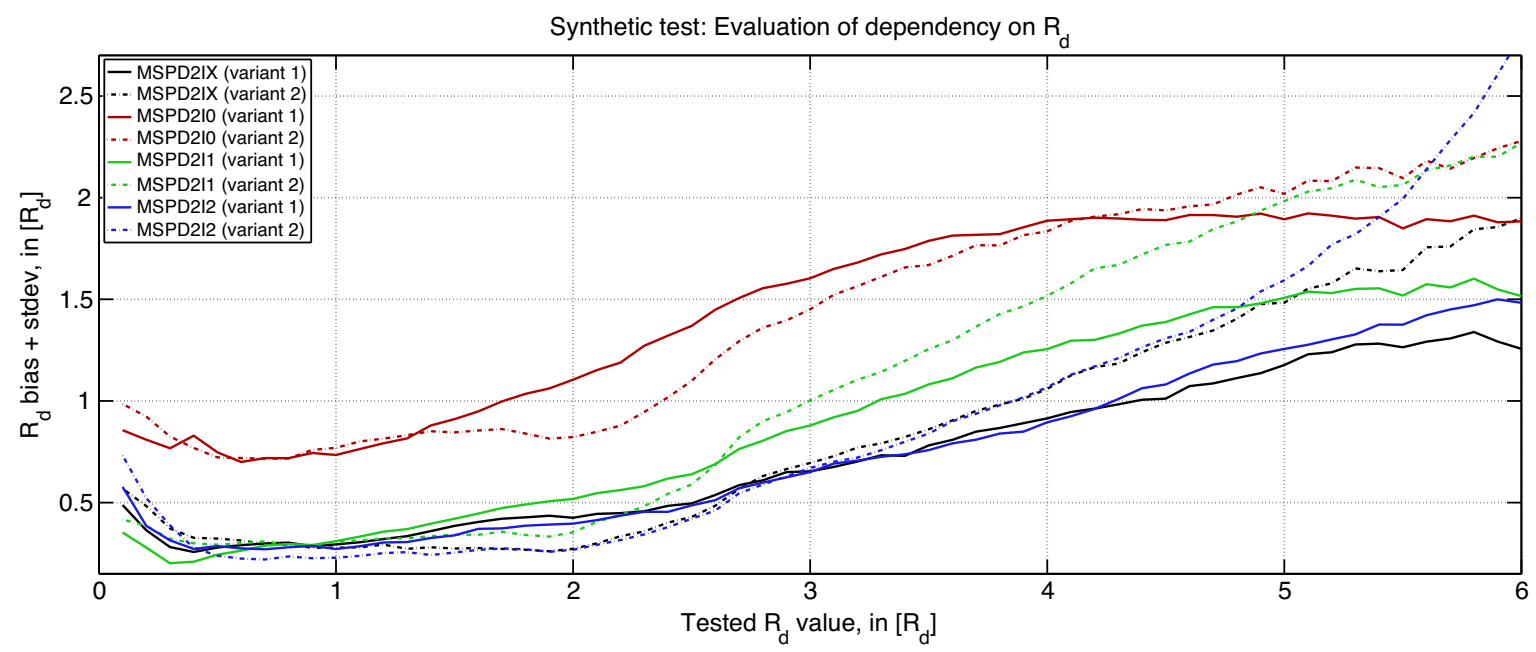

Fig. 5. Evaluation of $R_{d}$ estimation, dependency on voice quality measured in $R_{d}$. 


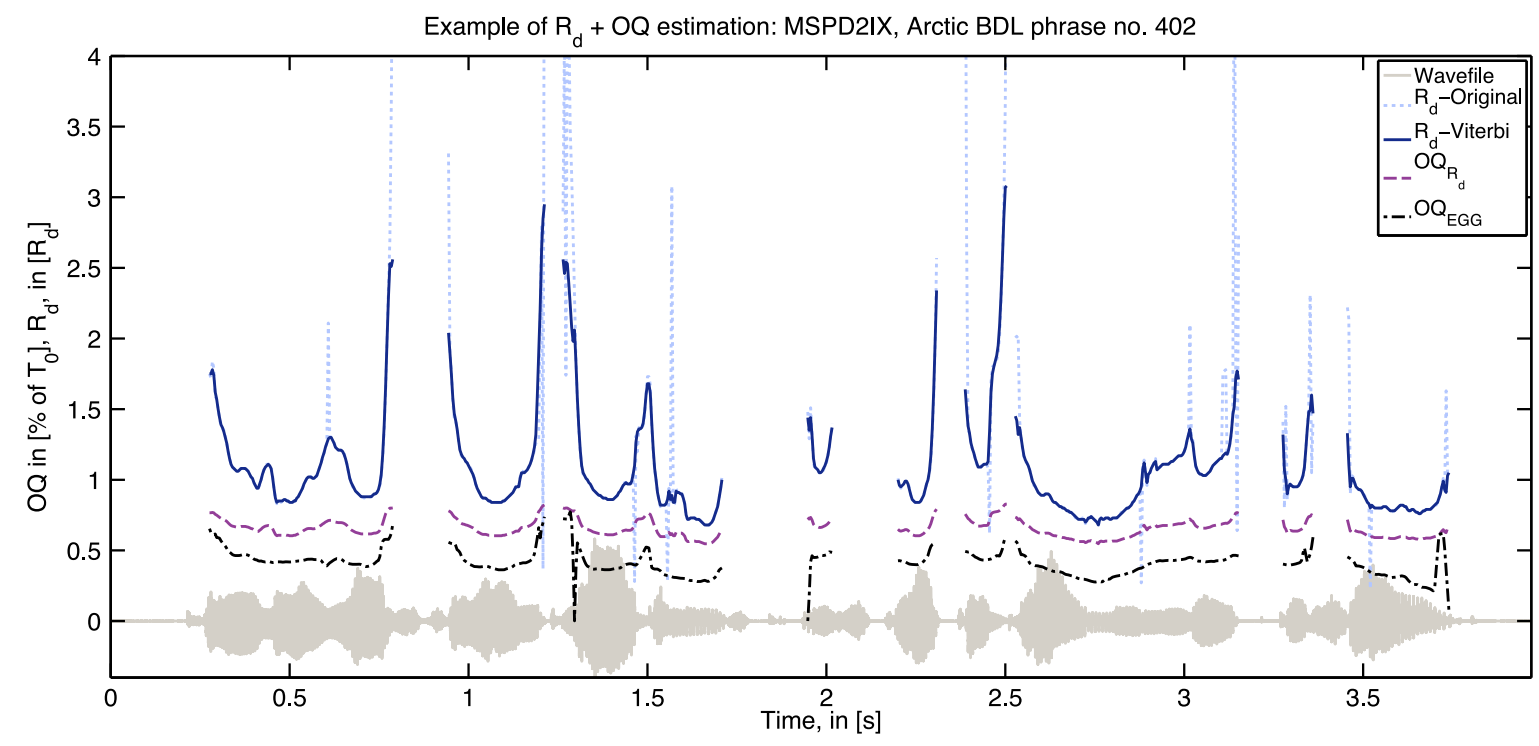

Fig. 6. Estimation example for method MSPD2IX, Viterbi smoothing, $\alpha=0.47$, CMU Arctic database, speaker BDL, phrase number 402.

\subsection{Test basis on EGG measurements}

No reliable ground truth is known to date to evaluate the estimation of glottal source parameters from natural human speech. A measurement of the movement of the glottal folds is given by EGG signals recorded simultaneously to speech signals. It is considered to form the basis of a more robust glottal source parameter estimation compared to estimations based on recorded audio signals of human speech. EGG waveforms are regarded as valid indicator of the vocal fold contact area to measure glottal activity (Baer et al., 1983). The differentiated EGG (DEGG) can be considered as reliable indicator of the time instant of glottal closing (GCI) (Henrich et al., 2004).

However, it is not yet validated that the glottal opening and closing events extracted from an EGG signal reflect exactly the time instants of the physiological contact of the vocal folds muscles (Baer et al., 1983; Childers et al., 1986; Orlikoff, 1991; Marasek, 1997). Furthermore, the EGG-based time instants may not exactly match the start and end of the glottal air flow (Fröhlich et al., 2001). Moreover, despite the general acceptance to provide more reliable estimates, the EGG-based measurements can still be inaccurate (Colton and Conture, 1990; Marasek, 1997; Sapienza et al., 1998). For example, a reliable and exact determination of the time instant of glottal opening (GOI) can be more difficult and erroneous (Baken, 1992; Baer et al., 1983) than the estimation of GCIs. The GOI estimation on EGG waveforms can especially be error-prone if strands of mucus bridge the glottis while the opening of the vocal folds (Titze and Talkin, 1979; Childers et al., 1986; Dromey et al., 1992). Other vocal fold vibratory motions than the modal register may lead as well to less robust estimations (Childers et al., 1986). The study of Herbst (2004) illustrates that each analyzed algorithm to estimate $O Q$ from an EGG signal introduces a bias, either by having to choose a certain threshold to measure the short-term peak-to-peak amplitudes of the EGG signal or by having to pick one of possibly several peaks from the DEGG signal appearing while the glottal opening phase (Childers and Lee, 1991).

Despite the mentioned problems we choose as test scenario for natural human speech the $O Q$ comparison using EGG and audio recordings because of its relatively easy setup and reasonable reliability in contrast to other methods. We compare the $O Q$ contours derived from each $R_{d}$ estimate $\left(O Q_{R_{d}}\right)$ with the $O Q$ contours estimated by the DECOM method (Henrich et al., 2004) on the corresponding EGG signals $\left(O Q_{E G G}\right)$. The example of Fig. 6 shows the curves of the frame-based $R_{d}$ estimator in light dotted, after Viterbi smoothing in dark solid, the from it derived $O Q_{R_{d}}$ contour in dashed and the $O Q_{E G G}$ reference in dash-dotted lines. We observe as in Childers and Lee (1991) and Herbst (2004) a general non-constant offset between the $O Q_{R_{d}}$ and $O Q_{E G G}$ contours due to the mentioned systematic bias of the $O Q$ estimation by the EGG-based technique. We choose the phrase shown in Fig. 6 to exemplify that the EGG measure can be error-prone as the physiologically impossible jumps of $O Q_{E G G}$ around 1.3 and $1.95 \mathrm{~s}$ illustrate. 
Error surface example of $R_{d}$ estimation for method MSPD2IX, BDL phrase 402

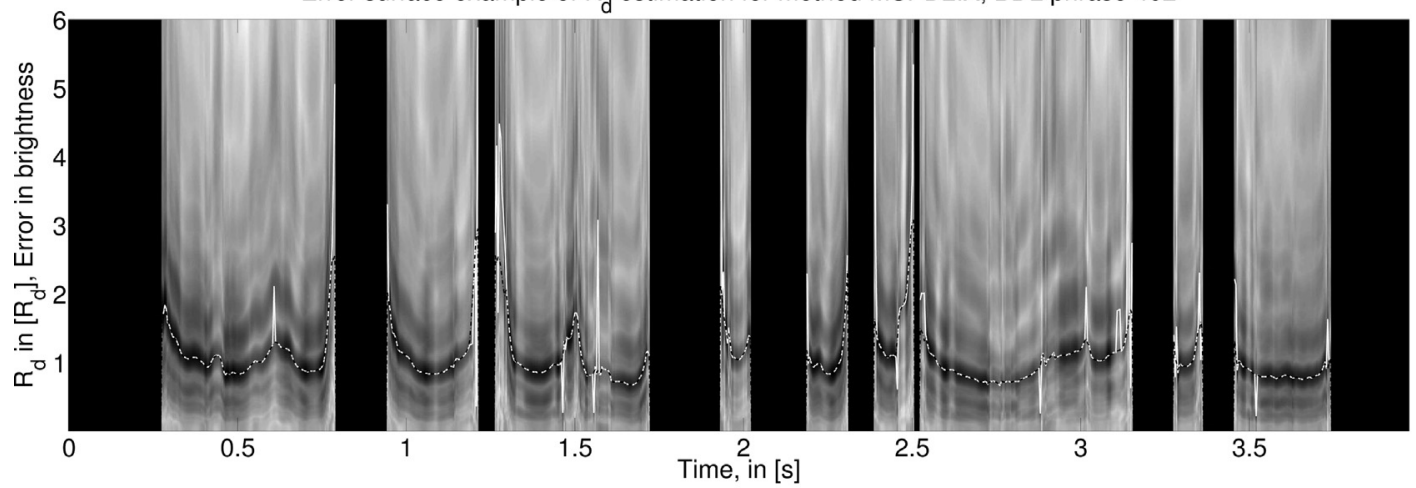

Error surface example of $R_{d}$ estimation for method MSPD2I0, BDL phrase 402

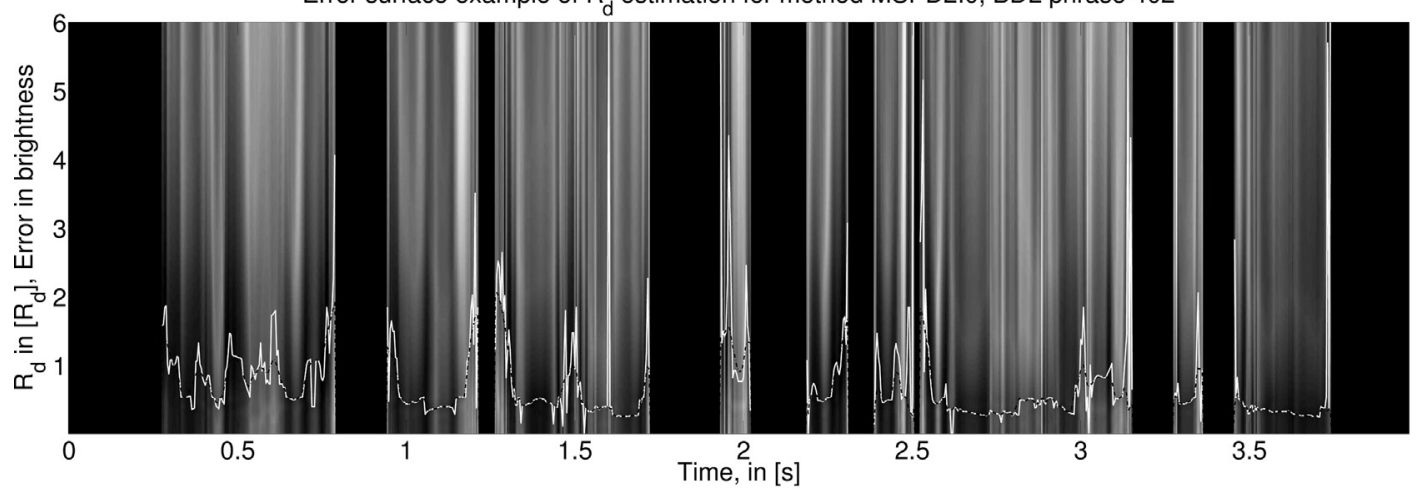

Error surface example of $R_{d}$ estimation for method MSPD2I1, BDL phrase 402

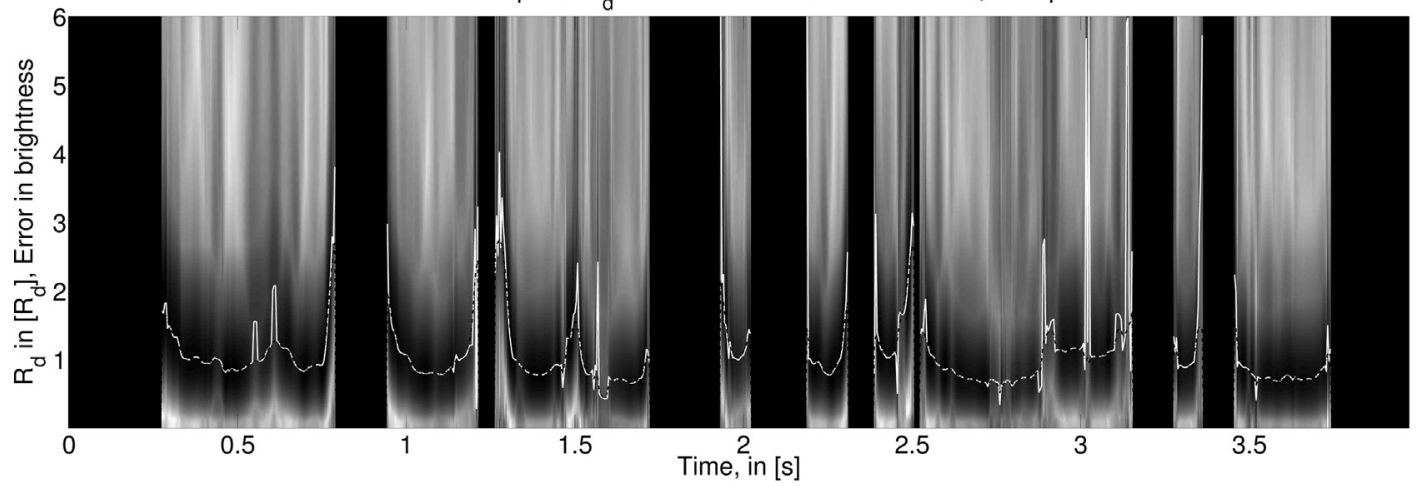

Error surface example of $R_{d}$ estimation for method MSPD2I2, BDL phrase 402

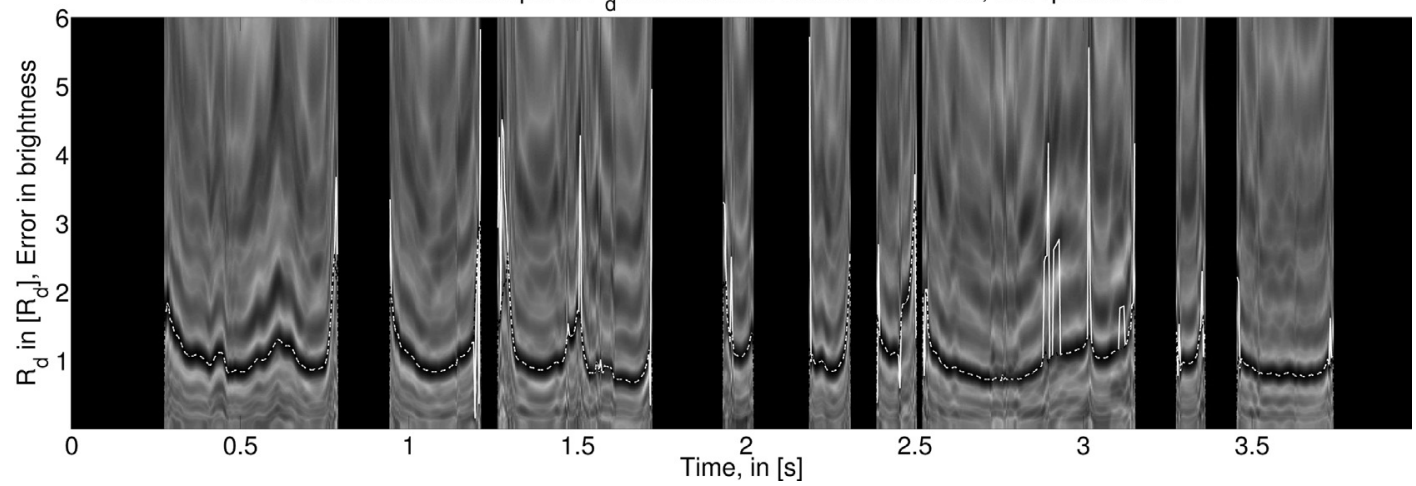

Fig. 7. $R_{d}$ error surface examples, 4 phase minimization methods, standard Viterbi smoothing, $\alpha=0.47$, BDL phrase 402 . 


\subsection{OQ test across speakers}

\subsubsection{Error surfaces of Viterbi smoothing}

Fig. 7 depicts examples of how Viterbi smoothing suppresses unnatural jumps of each frame-based $R_{d}$ estimator. The error residuals of the phase error functions of each phase minimization based objective functions generate an error curve per frame. Frames over time span up the illustrated error surfaces. Completely black segments are set as unvoiced. The error lattices of the voiced segments define the observation probability of the the noise-robust Viterbi algorithm. The Viterbi algorithm computes the highest probability which best explains the observation sequence $\mathrm{O}$ and which determines the optimal state sequence X per voiced segment. The optimal state sequences X of glottal source shapes $R_{d}$ of the standard Viterbi smoothing approach are illustrated as dashed gray lines. The initial $R_{d}$ estimates are illustrated in white colour and reflect the $R_{d}$ value where the frame-based phase error function exhibits the lowest error. Tiny error valleys in black around these initial $R_{d}$ estimates are very well developed for the methods MSPD2IX and MSPD2I2. MSPD2IX has distinctive error hills which are plotted with a brighter contrast and leaves little confusing side minima to its objective function to minimize the error of the phase error function. MSPD2I2 shares are similarly robust error surface for this natural speech example of speaker BDL. Side minima appear e.g. at $\sim 0.6 \mathrm{~s}$ at $R_{d} \approx 2.0$ and $R_{d} \approx 5.0$. Since these side minima are higher than the overall lowest error value, no unnatural jumps occur. The latter are present for MSPD2I 2 at $\sim 2.9 \mathrm{~s}$ where the initial $R_{d}$ estimate in white jumps three times from the apparently true $R_{d}$ contour and its obvious error valley at $R_{d} \approx 1.0$ to misleading side minima at $R_{d} \approx 2.5$ and $R_{d} \approx 4.0$. The method MSPD2I1 exhibits as well clear valleys which are broader and less distinctive than the ones of MSPD2IX and MSPD2I2. Its occurring side minima are more developed which results in a higher probability to produce physiological impossible jumps of the $R_{d}$ estimate. MSPD2I1 suffers for example at $\sim 0.6 \mathrm{~s}$ from a misleading side minima which got suppressed for MSPD2IX and MSPD2I2. The reason why the method MSPD2I0 performs worst is apparent when examining its error surface shown in Fig. 7. No clear error valleys in black for the underlying glottal excitation source contained in the analyzed speech phrase are established. Its original $R_{d}$ estimates in white and the smoothed $R_{d}$ contours in gray do not follow the true shape of the glottal source.

\subsubsection{Without Viterbi smoothing}

The Pearson product moment correlation coefficient $r$ (Pearson, 1900) normalizes the co-variance of $O Q_{R_{d}}$ and $O Q_{E G G}$ by the product of its standard deviations. We use $r$ as correlation metric to examine how well the $O Q_{R_{d}}$ derived from each $R_{d}$ estimate correlates with $O Q_{E G G}$. It is defined in the range $[-1,1]$ with -1 expressing a perfectly negative correlation, +1 a perfectly positive correlation, and 0 no correlation.

We use the root-mean-square error ( $r m s e$ ) as second evaluation metric. To avoid any impact of the bias that is present in the EGG-based $O Q$ estimate we remove the mean between the $O Q$ estimates for each voiced segment. Please note that the calculation of Pearsons product moment correlation coefficient $r$ implies the removal of a possible bias between both evaluated sample distributions. In the following, the Rd adaptation variants 1 and 2 from Section 5 are evaluated for each test scenario.

Table 1

$O Q$ test results, without Viterbi smoothing, Rd adaptation variant 1.

\begin{tabular}{lllll}
\hline & MSPD2IX & MSPD2I0 & MSPD2I1 & MSPD2I2 \\
\hline$r$ & 0.2958 & 0.1599 & 0.2910 & 0.3305 \\
rmse & 0.0835 & 0.1928 & 0.0804 & 0.0772 \\
\hline
\end{tabular}

Table 2

$O Q$ test results, without Viterbi smoothing, Rd adaptation variant 2 .

\begin{tabular}{lllll}
\hline & MSPD2IX & MSPD2I0 & MSPD2I1 & MSPD2I2 \\
\hline$r$ & 0.3189 & 0.1788 & 0.3138 & 0.3457 \\
rmse & 0.0765 & 0.1846 & 0.0747 & 0.0724 \\
\hline
\end{tabular}


Table 3

$O Q$ test results, median smoothing, order 5 .

\begin{tabular}{lllll}
\hline & MSPD2IX & MSPD2I0 & MSPD2I1 & MSPD2I2 \\
\hline$r$ & 0.3438 & 0.1776 & 0.3343 & 0.3711 \\
rmse & 0.0710 & 0.1336 & 0.0774 & 0.0635 \\
\hline
\end{tabular}

Table 4

Viterbi smoothing (optimal $\alpha$-values in parentheses).

\begin{tabular}{lllll}
\hline & MSPD2IX & MSPD2I0 & MSPD2I1 & MSPD2I2 \\
\hline$r$ & $0.5327(0.07)$ & $0.2404(0.13)$ & $0.4894(0.07)$ & $0.5241(0.09)$ \\
rmse & $0.0507(0.03)$ & $0.0564(0.01)$ & $0.0515(0.03)$ & $0.0513(0.01)$ \\
\hline
\end{tabular}

The results for each objective function estimating $R_{d}$ without applying Viterbi smoothing are listed in Tables 1 and 2. The method MSPD2I2 achieves the highest correlation and a smaller error between its estimated and the EGG-based $O Q$ contours. The results of MSPD2IX and MSPD2I1 are slightly worse. MSPD2I0 performs worst by a substantial margin. Please note that the baseline method MSPD2I1 of Degottex et al. (2011) constraint to the normal $R_{d}$ range [0.3, 2.7] and without Viterbi smoothing achieves $r=0.23$. By visual inspection its estimated $R_{d}$ and $O Q$ curves appear to fluctuate more. However, due to the constraint range the failures are less weighted. The $R_{d}$ adaptation variant 2 obtains better results for each method than variant 1 . Since variant 2 outperforms variant 1 for each test set on natural speech, we will only show $R_{d}$ adaptation variant 2 in the following.

\subsubsection{Smoothing with a moving average filter}

We examined different moving average filter types to evaluate their ability to suppress the local instabilities of each frame-based $R_{d}$ estimator that are present within short time segments. The best results were achieved by a median filter with order 5, shown in Table 3 . It improves the estimated $R_{d}$ contours of each phase minimization method only to a marginal extent. In the following we will investigate into means of establishing a more robust correction of the estimated $R_{d}$ contours by utilizing different configurations of the Viterbi algorithm.

\subsubsection{Standard Viterbi smoothing}

The results of Viterbi smoothing without the utilization of the novel $O Q_{G M M}$ prediction-based Viterbi steering are summarized in Table 4. The improvements of applying a dynamic programming algorithm to smooth the estimated glottal source shape curves are apparent when comparing its results with the ones of Tables 1 and 2 (without the application of Viterbi smoothing) and Table 3 (smoothing with a moving average filter). Especially the best performing methods MSPD2IX, MSPD2I2 and MSPD2I1 benefit enormously from Viterbi smoothing while the improvements for the worst method MSPD2IO are limited.

The $r$-correlation maxima and the rmse-error minima are shown per method. The corresponding $\alpha$-values to scale the observation probability of Viterbi smoothing are given in parentheses. One global maximum for the correlation $r$ and one global minimum for the error rmse exists for each method concerning the Viterbi parameter $\alpha$. The $r$-maxima occur for $\alpha$ in the range $[0.07,0.13]$ and lie with a maximal offset of $\alpha=0.10$ to the rmse-minima.

In the following we will present the results of the novel steering of Viterbi smoothing in the same manner. Each algorithm variant exhibits one global $r$-maxima and one global rmse-minima concerning the scaling parameters $\alpha$ or respectively $\beta$ of the Viterbi algorithm, introduced in Section 6. The objective of the following tests is to determine the best overall values a) for the scaling parameters $\alpha$ and $\beta$ of Viterbi smoothing and steering, and b) for each phase minimization method.

\subsubsection{Viterbi steering using GMM prediction}

We evaluate the GMM-based $O Q$ prediction by means of a 3-fold leave-one-out cross-validation on the training and test sets corresponding to each speaker database. The rmse-error and the $r$-correlation of each $O Q_{G M M}$ prediction model using the feature set of model 1 are shown in Table 5 and respectively the feature set of model 2 in Table 6. 
Table 5

Validation on training and test sets per speaker, model 1.

\begin{tabular}{lllll}
\hline & rmse $_{\text {training }}$ & rmse $_{\text {test }}$ & $r_{\text {training }}$ & $r_{\text {test }}$ \\
\hline BDL & 0.0837 & 0.1140 & 0.5508 & 0.4705 \\
JMK & 0.0837 & 0.0787 & 0.7280 & 0.3500 \\
SLT & 0.0574 & 0.1114 & 0.9054 & 0.3956 \\
\hline
\end{tabular}

Table 6

Validation on training and test sets per speaker, model 2.

\begin{tabular}{lllll}
\hline & rmse $_{\text {training }}$ & rmse $_{\text {test }}$ & $r_{\text {training }}$ & $r_{\text {test }}$ \\
\hline BDL & 0.0781 & 0.0592 & 0.6273 & 0.7285 \\
JMK & 0.0742 & 0.0917 & 0.7926 & 0.3478 \\
SLT & 0.0529 & 0.1072 & 0.9209 & 0.4030 \\
\hline
\end{tabular}

Table 7

Viterbi steering, model 1 (optimal $\alpha$-values in parentheses).

\begin{tabular}{lllll}
\hline & MSPD2IX & MSPD2I0 & MSPD2I1 & MSPD2I2 \\
\hline$r$ & $0.5348(0.07)$ & $0.4202(0.05)$ & $0.5047(0.09)$ & $0.5227(0.03)$ \\
$r m s e$ & $0.0502(0.05)$ & $0.0536(0.07)$ & $0.0510(0.07)$ & $0.0507(0.03)$ \\
\hline
\end{tabular}

Please note that by the straightforward training of a GMM using the $O Q_{E G G}$ contours and the corresponding voice descriptor feature combination of two speakers, the predicted $O Q_{G M M}$ contours for the test set on the third speaker achieves correlations for $r_{\text {test }}$ shown in Tables 5 and 6 being close to the performance of the signal processing based $R_{d}$ estimation methods. The strong potential of the proposed $O Q$ prediction using voice descriptors is indicated by the corresponding results on the training test set $r_{\text {training }}$ which by far outperform the results discussed in the following tests. However, the remaining principal problem is to overcome the speaker-dependency of the modelling to generalize better over speaker specific characteristics. This could be solved by employing more speaker databases and a more sophisticated handling of the feature combination.

The higher prediction accuracies of the training versus the test sets for the speakers JMK and SLT indicate that the utilized feature sets do not generalize optimally on their data sets. However, the $O Q_{G M M}$ prediction model 2 for speaker BDL is able to predict more precise $O Q_{G M M}$ contours on the BDL test set than on its own training set of the speakers JMK and SLT. In Section 8.4 we will analyze more in detail the intrinsic characteristics of each speaker data set of the utilized CMU Arctic databases by examining the $r$-correlation and rmse-error results per speaker.

In the following sections we will examine the utilization of the models trained to evaluate the test set errors. This reflects the later application of the proposed novel Viterbi steering to estimate $R_{d}$ where no training data will be available.

\section{(a) Viterbi steering, $O Q_{G M M}$ prediction model 1}

The results of the four phase minimization methods using Viterbi smoothing and its auxiliary GMM prediction based Viterbi steering for model 1 of Section 6 are shown in Table 7.

For this test we vary the Viterbi scaling parameter $\alpha$ while fixing the Viterbi scale $\beta$ to a constant value of 1.0. The values in parentheses illustrate the $\alpha$ values of the $r$-maxima and rmse-minima. A one-way ANOVA comparison (Hill and Lewicki, 2007) with the correlation results of standard Viterbi smoothing presented in the Table 4 validates that the improvements of Viterbi steering using model 1 are statistically significant for method MSPD2I1 at significance level $1 \%$ ( $p$-value $<0.01$ ) and for method MSPD2I0 at significance level $0.1 \%$ ( $p$-value $<0.001)$. No statistically significant improvements could be validated for the methods MSPD2IX and MSPD2I2.

A one-way ANOVA analysis of the corresponding optimization of the scale parameter $\beta$ for Viterbi steering demonstrated that $\beta$ has no statistically significant influence on each of the evaluated phase minimization variants.

(b) Viterbi steering, $O Q_{G M M}$ prediction model 2 
Table 8

Viterbi steering, model 2 (optimal $\alpha$-values in parentheses).

\begin{tabular}{lllll}
\hline & MSPD2IX & MSPD2I0 & MSPD2I1 & MSPD2I2 \\
\hline$r$ & $0.5437(0.05)$ & $0.4623(0.01)$ & $0.5234(0.05)$ & $0.5337(0.01)$ \\
rmse & $0.0498(0.03)$ & $0.0515(0.03)$ & $0.0501(0.05)$ & $0.0499(0.01)$ \\
\hline
\end{tabular}

Table 9

Viterbi steering, model 2 (optimal $\beta$-values in parentheses).

\begin{tabular}{lllll}
\hline & MSPD2IX & MSPD2I0 & MSPD2I1 & MSPD2I2 \\
\hline$r$ & $0.5438(1.06)$ & $0.4625(1.03)$ & $0.5246(0.76)$ & $0.5343(0.76)$ \\
rmse & $0.0498(1.50)$ & $0.0502(0.20)$ & $0.0501(1.00)$ & $0.0499(1.09)$ \\
\hline
\end{tabular}

To further augment the robustness of the glottal source shape parameter estimation on natural speech we use as additional voice descriptor the fundamental frequency $F_{0}$ for the $O Q_{G M M}$ model 2 . A comparison of each rmse-error and each $r$-correlation value for each speaker between Table 5 (model 1, without $F_{0}$ ) and Table 6 (model 2, with $F_{0}$ ) shows that the consideration of $F_{0}$ contributes to the robustness of the GMM estimation model to predict $O Q_{G M M}$. On the one hand, only the correlation on the data test set for speaker JMK deteriorates to a marginal extent from $r=0.3500$ for model 1 to $r=0.3478$ for model 2. On the other hand, the correlation on the data test set for speaker BDL augments by employing $F_{0}$ from $r=0.4705$ for model 1 to $r=0.7285$ for model 2 to a significant extent.

Again, we fix the scale parameter $\beta$ to 1.0 and vary the scale parameter $\alpha$. Table 8 illustrates the results of the $\alpha$-optimization for the Viterbi steering of model 2. We execute the one-way ANOVA analysis between the results of the corresponding test to optimize the scale parameter $\alpha$ of Viterbi steering using model 1 (illustrated in Table 7) with Viterbi steering using model 2 (listed in Table 8). It demonstrates improvements to a statistically significant extent for method MSPD2I2 at significance level 5\% ( $p$-value $<0.05$ ), for method MSPD2I1 at significance level $1 \%(p$ value $<0.01$ ), and for method MSPD2I0 again at significance level $0.1 \%$ ( $p$-value $<0.001$ ). No statistically significant improvement could be measured for the overall best performing method MSPD2IX when using model 2 compared to using model 1. Please note that the $r$-correlation improved for MSPD2IX slightly from $r=0.5348$ (listed in Table 7) to $r=0.5437$ (listed in Table 8).

Fixing the determined $\alpha$-maxima in terms of the measured $r$-correlations, depicted in Table 8 , to optimize the scale parameter $\beta$, whose results are illustrated in Table 9, does not exhibit statistically significant improvements. We show the $\beta$-optimization of Table 9 depicting the overall best performance of the $O Q$ comparison test to motivate the following one-way ANOVA analysis. It validates that applying Viterbi steering is statistically significant for all evaluated phase minimization variants. We compare the evaluation distributions of standard Viterbi smoothing (illustrated in Table 4) with the overall best Viterbi steering results, the $\beta$-optimization of model 2 (shown in Table 9). The improvements are statistically significant for method MSPD2IX at significance level 5\% ( $p$-value $<0.05$ ), for method MSPD2I2 at significance level $10 \%$ ( $p$-value $<0.1$ ), as well as for the methods MSPD2I1 and MSPD2I0 at significance level $0.1 \%$ ( $p$-value $<0.001)$.

\section{(c) Viterbi steering summary}

The utilization of the novel Viterbi steering approach to augment the robustness of Viterbi smoothing when applied to smooth the contours of the estimated glottal source shape parameters demonstrates improvements to a statistically significant extent when comparing the results with standard Viterbi smoothing a) for both employed GMM models, b) for each of the four $R_{d}$ estimation methods and c) for both $R_{d}$ regression adaptation variants. Please note that we did only discuss the results for $R_{d}$ regression adaptation variants 2 while variant 1 proved to be as well statistically significant.

The usage of the $O Q_{G M M}$ prediction model 2 outperforms model 1 for each $R_{d}$ estimator and each $R_{d}$ regression variant on each evaluation metric ( $r$-correlation and rmse-error). This suggests that the fundamental frequency $F_{0}$ as the first dimension of prosody (Fant and Liljencrants, 1981) not just correlates with the contours of parameterized glottal excitation source shapes but can be exploited as covariation feature additionally to the other proposed voice descriptors to estimate glottal source parameters. However, this has to be evaluated on a bigger test set employing more speakers to evaluate the speaker-dependency. 
Table 10

Comparison results of other methods.

\begin{tabular}{llll}
\hline & DyProg-LF & Strik-LF & PowRd \\
\hline$r$ & 0.3721 & 0.1215 & 0.1776 \\
rmse & 0.0716 & 0.1217 & 0.1760 \\
\hline
\end{tabular}

The $R_{d}$ regression adaptation variant 2 achieves the overall better results compared to variant 1 on the $O Q$ comparison test. The overall best performing $R_{d}$ estimation method is MSPD2IX, followed by MSPD2I2, MSPD2I1 and respectively MSPD2I0. It was shown that the worst performing method MSPD2I0 profits the most from the auxiliary steering of the Viterbi algorithm while the best performing method MSPD2IX profits the least from Viterbi steering. On the one hand, this suggests that the $O Q_{G M M}$ prediction exploiting the covariation of other voice descriptors is a relatively robust manner to be used for glottal source parameter estimation. On the other hand, this conclusion indicates that MSPD2IX and to some extent the good performing methods MSPD2I2 and MSPD2I1 may already estimate comparatively robust $R_{d}$ curves by the utilization of standard Viterbi smoothing without the additional steering. As discussed in Section 8.2 and shown with Fig. 6, the EGG-based technique is not error-free. The employed evaluation metric is therefore limited and prevents the potential to achieve higher correlation and lower error measurements.

\subsection{OQ test of other methods across speakers}

We examine the results of the following other glottal source estimation algorithms on the same test set of the CMU Arctic databases to provide an objective comparison to our algorithm variants by employing the same evaluation metric. We denominate the method introduced in Kane et al. (2012), Kane and Gobl (2013a) as DyProg-LF. It is based on first estimating GCI locations using a modified version of the SE-DREAMS approach of Drugman et al. (2012b) described in Kane and Gobl (2013a). Then the iterative adaptive inverse filtering method (Alku, 1992) is used to compute the glottal source signals on which error values are calculated. The error criteria are based on measuring the correlation of synthesized LF model pulses parameterized over a grid of $R_{d}$ values and matched to the source signals in the spectral and the time domain. The temporal and spectral errors form a target cost. The continuity of the parameter trajectories over frames forms a transition cost. Both costs are utilized in a dynamic programming algorithm to increase the robustness of the DyProg-LF algorithm to estimate the $R_{d}$ contour of the glottal excitation source over time.

The second method chosen for comparison is called Strik-LF, proposed in Strik et al. (1993), Strik (1998). For this work the same glottal source signals estimated by the inverse filtering method of the DyProg-LF algorithm were utilized. The method estimates LF model parameters and its amplitude measures directly on glottal source signals in the time domain. A two part optimization procedure improves the LF model parameter estimation. First, the Nelder and Mead simplex optimization algorithm is applied being insensitive to large errors in the initialization. Second, a steepest descent optimization algorithm further refines the LF model fit.

As third method we evaluate the power spectrum based method of Ó Cinnéide (2012) called PowRd. To determine $R_{d}$ it avoids unreliable phase information and high frequency information which can be corrupted by noise. A relative Itakura Saito error criterion determines the filter order and the coefficients of the vocal tract filter as well as the scale parameter $E_{e}$ of the included LF voice source model. The PowRd method is based on the SIM approach of Fröhlich et al. (2001) and is thus as well robust to phase disturbances and the window position of the analyzed frame with respect to the period in time.

For all three methods we measure as well a non-constant offset between the $O Q$ curves estimated from the audio recordings versus the $O Q$ curves derived from the corresponding EGG recordings. It confirms the observation of a possible systematic $O Q$ bias by the EGG-based method discussed in Section 8.1.

However, since the author of Kane and Gobl (2013a) provided the $O Q$ estimation results for the methods StrikLF and DyProg-LF using the method described in Drugman et al. (2012b) to estimate the required GCIs, the GCI time instants and voicing decisions were not completely congruent to our DECOM basis, leading to a slightly different evaluation metric. The Strik-LF and the DyProg-LF method are based on the same estimation of the glottal source signal from inverse filtering. The better performance of the DyProg-LF compared to the Strik-LF method shown in Table 10 confirms to a certain extent the conclusions we drew from our results. Dynamic programming immensely improves the results of glottal source shape parameter estimation by suppressing unnatural jumps in short-time segments. On 
Table 11

Mean $\mu$ and standard deviation $\sigma$ of speaker characteristics.

\begin{tabular}{lccc}
\hline & BDL & JMK & SLT \\
\hline$F_{0_{\mu}}$ & $121.83 \mathrm{~Hz}$ & $112.42 \mathrm{~Hz}$ & $174.19 \mathrm{~Hz}$ \\
$F_{0_{\sigma}}$ & $17.16 \mathrm{~Hz}$ & $13.91 \mathrm{~Hz}$ & $17.38 \mathrm{~Hz}$ \\
$O Q_{E G G_{\mu}}$ & 0.41 & 0.62 & 0.54 \\
$O Q_{E G G_{\sigma}}$ & 0.09 & 0.07 & 0.10 \\
\hline
\end{tabular}

the other hand, parts of the better performance of the DyProg-LF method can be assigned to the conjoint optimization in the time and the spectral domain.

The evaluated variant of the PowRd method is frame-based without the utilization of a dynamic programming approach. Its estimation robustness could therefore be augmented by employing as well a smoothing algorithm.

\subsection{OQ test per speaker}

The preceding sections of the $O Q$ comparison test illustrated the performance of the employed algorithms generalized over different speakers to estimate $R_{d}$. However, it is of vital interest to examine the estimation robustness of each method in dependency to the intrinsic peculiarities of each speaker.

Table 11 shows the mean $\mu$ and standard deviation $\sigma$ for the fundamental frequency $F_{0}$ and the $O Q$ derived from the EGG signals, measured on all voiced segments and all phrases for each speaker. The two male speakers BDL and JMK exhibit a comparatively lower mean pitch $F_{0_{\mu}}$ than the female speaker SLT. Speaker JMK has the least variance $\sigma^{2}$ in his $F_{0}$ contour while SLT and BDL exhibit larger $F_{0}$ variations. This observation for BDL corroborates the findings from Drugman et al. (2012). JMK demonstrates the highest mean open quotient $O Q_{E G G}$ with the lowest variance $\sigma^{2}$.

From informal listening tests we perceive that BDL has a very clear articulation and speaks with a high vocal effort. BDL has an overall modal phonation but uses quite often creaky voice offsets (Drugman et al., 2012) which may degrade the $R_{d}$ estimation accuracy due to the non-modal phonation of the creaky voice quality. JMK has a clear articulation but speaks with a weak vocal effort which partially results in a whispered (Obin, 2012) and creaky voice quality (Titze, 1994) with a bit of nasality. SLT under-articulates and speaks with a low vocal effort. She has a rather modal phonation with a bit of nasality. All three speakers talk with a low pulmonic pressure (Catford, 1977). BDL and to a less extent STL exhibit a pressed voice quality. JMK in contrary has a more relaxed voice quality which can lead according to the evaluation of the synthetic test set in Section 7 to a less good glottal source shape parameter estimation performance.

In the following sections we will examine the $O Q$ comparison test results per speaker. We will only show the results for each speaker using $R_{d}$ adaptation variant 2 which demonstrates the better performance throughout the whole $O Q$ comparison test. To restrict the huge amount of test results conducted for this study we only discuss important findings based on Pearsons correlation metric $r$.

The values which will be given in the following sections to each correlation $r$ in parenthesis correspond to the following indices of each algorithmic variant:

1 Without Viterbi smoothing.

2 Standard Viterbi smoothing.

3 Viterbi steering, model $1, \alpha$ variation, $\beta$ fixation.

4 Viterbi steering, model $2, \alpha$ variation, $\beta$ fixation.

\subsubsection{OQ test results for speaker $B D L$}

The results of the synthetic test discussed in Section 7 associate higher $F_{0}$ values as well as higher $R_{d}$ and $O Q$ values with a lower performance in estimating glottal source shape parameters. Speaker BDL presents among the three evaluated speakers the highest $r$-correlations, listed in Table 12. He has the lowest mean open quotient $O Q_{E G G_{\mu}}$ and a comparatively low $F_{0}$. His high vocal effort and clear articulation contribute to the ease of estimating his glottal excitation source shape. The utilization of $F_{0}$ within the voice descriptor set for prediction model 2 contributes to the 
Table 12

BDL $r$-correlation results ( $\alpha$ - or $\beta$-values in parentheses).

\begin{tabular}{lllll}
\hline & MSPD2IX & MSPD2I0 & MSPD2I1 & MSPD2I2 \\
\hline$r(1)$ & 0.5165 & 0.4761 & 0.5005 & 0.5233 \\
$r(2)$ & $0.7263(0.05)$ & $0.6029(0.21)$ & $0.6820(0.03)$ & $0.7043(0.11)$ \\
$r(3)$ & $0.7312(0.07)$ & $0.6323(0.13)$ & $0.6989(0.09)$ & $0.7021(0.03)$ \\
$r(4)$ & $0.7771(0.01)$ & $0.7479(0.03)$ & $0.7708(0.03)$ & $0.7568(0.01)$ \\
\hline
\end{tabular}

Table 13

BDL comparison results of other methods.

\begin{tabular}{llll}
\hline & DyProg-LF & Strik-LF & PowRd \\
\hline$r$ & 0.6606 & 0.3268 & 0.3315 \\
\hline
\end{tabular}

Table 14

JMK $r$-correlation results ( $\alpha$ - or $\beta$-values in parentheses).

\begin{tabular}{llcll}
\hline & MSPD2IX & MSPD2I0 & MSPD2I1 & MSPD2I2 \\
\hline$r(1)$ & 0.1902 & -0.0183 & 0.1755 & 0.2478 \\
$r(2)$ & $0.4267(0.05)$ & $0.0322(0.05)$ & $0.3922(0.07)$ & $0.4235(0.03)$ \\
$r(3)$ & $0.4299(0.03)$ & $0.3307(0.01)$ & $0.3900(0.07)$ & $0.4242(0.01)$ \\
$r(4)$ & $0.4344(0.05)$ & $0.2646(0.01)$ & $0.3937(0.09)$ & $0.4274(0.03)$ \\
\hline
\end{tabular}

Table 15

JMK comparison results of other methods.

\begin{tabular}{llll}
\hline & DyProg-LF & Strik-LF & PowRd \\
\hline$r$ & 0.0879 & -0.1106 & 0.0797 \\
\hline
\end{tabular}

$R_{d}$ estimation robustness for speaker BDL. The other three methods DyProg-LF, Strik-LF, and PowRd achieve as well the best $R_{d}$ estimation results for speaker BDL, illustrated in Table 13.

\subsubsection{OQ test results for speaker JMK}

Speaker JMK poses not before expected problems to estimate the shape of his glottal excitation source. Despite the lowest mean $F_{0}$ of all speakers his high mean open quotient $O Q_{E G G_{\mu}}$ measured and his weak vocal effort perceived lead to less good $R_{d}$ estimation results. By informal visual inspection of the $O Q_{E G G}$ contours for speaker JMK we realize that the EGG-based $O Q$ reference exhibits as well more physiological impossible movements compared to the other speakers $O Q_{E G G}$.

The Viterbi steering of model 2 using $F_{0}$ demonstrates only slight improvements for all but the worst performing method MSPD2I0, depicted in Table 14. The latter does not benefit from the exploitation of the $F_{0}$-covariation but from the utilization of Viterbi steering in general.

Moreover, the three methods employed for comparison have even greater problems to establish performant $R_{d}$ estimation results for speaker JMK, shown in Table 15. The PowRd method without the utilization of dynamic programming achieves a similar performance to the DyProg-LF approach using dynamic programming.

\subsubsection{OQ test results for speaker $S L T$}

The $R_{d}$ estimation results lie for speaker SLT with the proportionally highest $F_{0_{\mu}}$ in the range measured for speaker JMK.

No method could benefit from $F_{0}$ as voice descriptor for the $O Q_{G M M}$ prediction model 2, shown in Table 16. It indicates that the rather large difference in $F_{0_{\mu}}$ between the employed training data set of the male speakers BDL 
Table 16

SLT $r$-correlation results ( $\alpha$ - or $\beta$-values in parentheses).

\begin{tabular}{lllll}
\hline & MSPD2IX & MSPD2I0 & MSPD2I1 & MSPD2I2 \\
\hline$r(1)$ & 0.2485 & 0.0764 & 0.2636 & 0.2651 \\
$r(2)$ & $0.4486(0.15)$ & $0.0943(0.53)$ & $0.4005(0.13)$ & $0.4541(0.11)$ \\
$r(3)$ & $0.4497(0.15)$ & $0.3929(0.03)$ & $0.4280(0.15)$ & $0.4500(0.11)$ \\
$r(4)$ & $0.4444(0.15)$ & $0.3772(0.03)$ & $0.4197(0.13)$ & $0.4454(0.11)$ \\
\hline
\end{tabular}

Table 17

SLT comparison results of other methods.

\begin{tabular}{llll}
\hline & DyProg-LF & Strik-LF & PowRd \\
\hline$r$ & 0.3638 & 0.1206 & 0.1204 \\
\hline
\end{tabular}

and JMK versus the test data set for the female speaker SLT requires more speaker data with higher pitch to train the prediction model 2 using $F_{0}$.

The DyProg-LF method listed in Table 17 benefits from the application of its used dynamic programming approach and achieves nearly a similar performance compared to the phase minimization variants using Viterbi smoothing and steering.

\section{Summary and conclusions}

\subsection{Summary}

In this paper we proposed a novel technique we denominate Viterbi steering to aid the estimation of glottal source shape parameters. It is based on exploiting the covariation of other voice descriptors to steer and optimize the glottal source estimation using Viterbi smoothing. This work presents additionally an in-depth analysis of glottal source estimation using phase minimization variants by discussing the results of two extensive objective evaluation tests on synthetic and natural human speech signals. Moreover, two variants to adapt and extent the range of the glottal source shape parameter $R_{d}$ to parameterize the LF glottal source model are discussed.

\subsection{Conclusions}

From the works presented in this study we draw the following conclusions: It has been shown that the exploitation of covariation voice features is able to increase the robustness of the estimation of glottal excitation source parameters describing the voice quality of human speech for the employed data set. The utilization of a machine learning approach to classify voice qualities is able to aid and to possibly outperform the signal processing paradigms known to date to estimate glottal source signals in the future, if implemented in a more sophisticated manner. For example, the voice descriptor $F_{0}$ for the $O Q_{G M M}$ prediction model 2 was utilized without a possible normalization by its mean $F_{0_{\mu}}$ per speaker. It could especially render for speaker SLT with the highest $F_{0_{\mu}}$ a better estimation performance for Viterbi steering of model 2. Also, the performance of the novel Viterbi steering approach using the GMM-based $R_{d}$-predictor should improve by the utilization of a bigger database covering more speaker characteristics in the trained models. Moreover, additional voice quality features as in Kane and Gobl (2013b), the consideration of an intensity measure like a relative SPL originated form the subglottal pressure (Laver, 1968; Vilkman et al., 1999; Fant and Kruckenberg, 2005) or the Harmonic Richness Factor (HRF) (Childers and Lee, 1991) to reflect a higher sinusoidal signal content related to tense voices and a higher noise signal content related to breathy voices should contribute to the $O Q_{G M M}$ prediction robustness. The importance to smooth estimated glottal source parameters over time was validated as in Vincent et al. (2007), Kane et al. (2012) and Kane and Gobl (2013b) by the experimental findings. The proposed steering and the utilization of standard Viterbi smoothing demonstrates improvements to a statistically significant extent. The methods MSPD2I2 and MSPD2IX proposed in our last study (Huber et al., 2012) perform better than their corresponding baseline approach MSPD2I1 established in the preceding works (Degottex et al., 2010, 2011; Degottex, 2010). The 
estimation of voice qualities with a comparatively higher relaxed phonation and less vocal effort poses more difficulties. The $R_{d}$ adaptation variant 2 proposed in this study showed to provide a more precise distinction between glottal source shapes for the normal $R_{d}$ range. This can be the reason for its better performance on the test set of natural human speech where values of $R_{d}$ occur predominantly in the normal $R_{d}$ range.

\section{Acknowledgements}

The main author is supported by CIFRE contract no. 2011/2011, a collaboration between the research institute IRCAM and the company Acapela Group. He would like to thank Dr. John Kane for the preparation of the test results for the DyProg-LF and Strik-LF method as well as for the kind feedback and discussion concerning the utilized speaker databases. Furthermore, he would like to thank Dr. Alan Ó Cinnéide for the disposal of and the kind help on his PowRd method. Also, thanks go to Dr. Olga Gordeeva from the Acapela Group for the analysis of the speaker databases. Finally, he is very grateful for the kind attendance by his supervisor Dr. Axel Röbel.

\section{References}

Alku, P., 1992. Glottal wave analysis with pitch synchronous iterative adaptive inverse filtering. Speech Communication 11 (2-3), $109-118$.

Alku, P., Bäckström, T., Vilkman, E., 2002. Normalized amplitude quotient for parametrization of the glottal flow. Journal of the Acoustical Society of America 112 (2), 701-710.

Alku, P., Vilkman, E., 1996. Amplitude domain quotient for characterization of the glottal volume velocity waveform estimated by inverse filtering. Speech Communication 18 (2), 131-138.

Baer, T., Lofqvist, A., McGarr, N., 1983. Laryngeal vibrations: A comparison between high-speed filming and glottographic techniques. Journal of the Acoustical Society of America 73 (4), 1304-1308

Baken, R.J., 1992. Electroglottography. Journal of Voice 6 (2), 98-110.

Catford, J.C., 1977. Fundamental Problems in Phonetics. Indiana University Press.

Chen, G., Samlan, R.A., Kreiman, J., Alwan, A., 2013. Investigating the relationship between glottal area waveform shape and harmonic magnitudes through computational modeling and laryngeal high-speed videoendoscopy. In: 14th Annual Conference of the International Speech Communication Association (Interspeech ICSLP), Lyon, France.

Childers, D.G., Hicks, D.M., Moore, G.P., Alsaka, Y.A., 1986. A model for vocal fold vibratory motion, contact area, and the electroglottogram. Journal of the Acoustical Society of America 80 (5), 1309-1320.

Childers, D.G., Lee, C.K., 1991. Vocal quality factors: analysis, synthesis, and perception. Journal of the Acoustical Society of America 90 (5), 2394-2410.

Colton, R.H., Conture, E.G., 1990. Problems and pitfalls of electroglottography. Journal of the Voice Foundation 4 (1), $10-24$.

Degottex, G., 2010. Glottal source and vocal tract separation. IRCAM, Paris (PhD thesis).

Degottex, G., Roebel, A., Rodet, X., 2010. Joint estimate of shape and time-synchronization of a glottal source model by phase flatness. In: Proceedings of the IEEE International Conference on Acoustics, Speech, and Signal Processing (ICASSP), Dallas, USA, pp. 5058-5061.

Degottex, G., Roebel, A., Rodet, X., 2011. Phase minimization for glottal model estimation. IEEE Transactions on Acoustics, Speech and Language Processing 19 (5), 1080-1090.

Doval, B., d'Alessandro, C., Henrich, N., 2006. The spectrum of glottal flow models. Acta Acustica united with Acustica 92 (6), $1026-1046$.

Dromey, C., Stathopoulos, E.T., Sapienza, C.M., 1992. Glottal airflow and electroglottographic measures of vocal function at multiple intensities. Journal of Voice 6 (1), 44-54.

Drugman, T., Dubuisson, T., Moinet, A., d'Alessandro, N., Dutoit, T., 2008. Glottal source estimation robustness - a comparison of sensitivity of voice source estimation techniques. In: IEEE International Conference on Signal Processing and Multimedia Applications (SIGMAP), Porto, Portugal, pp. 202-207.

Drugman, T., Kane, J., Gobl, C., 2012. Modeling the creaky excitation for parametric speech synthesis. In: 13th Annual Conference of the International Speech Communication Association (Interspeech ICSLP), Portland, Oregon, USA.

Drugman, T., Thomas, M., Gudnason, J., Naylor, P., Dutoit, T., 2012b. Detection of glottal closure instants from speech signals: a quantitative review. IEEE Transactions on Audio, Speech, and Language Processing 20 (3), 994-1006.

Ellis, D.P.W., 2005. PLP and RASTA (and MFCC, and inversion) in Matlab, online web resource: http://www.ee.columbia.edu/dpwe/ resources/matlab/rastamat/.

Fant, G., 1995. The LF-model revisited. Transformation and frequency domain analysis. In: Quarterly Progress and Status Report, vol. 36, no. 2-2. Department of Speech, Music and Hearing, KTH, pp. 119-156.

Fant, G., 1997. The voice source in connected speech. Speech Communication 22 (2-3), 125-139.

Fant, G., Kruckenberg, A., 2005. Covariation of subglottal pressure f0 and intensity. In: Interspeech 2005 - Eurospeech, 9th European Conference on Speech Communication and Technology, Lisbon, Portugal, pp. 1061-1064.

Fant, G., Kruckenberg, A., Liljencrants, J., Båvegård, M., 1994. Voice source parameters in continuous speech. Transformation of LF-parameters. In: 3rd International Conference on Spoken Language Processing (ICSLP), Yokohama, Japan, pp. 1451-1454. 
Fant, G., Kruckenberg, A., Liljencrants, J., Båvegård, M., 2000. Acoustic-phonetic studies of prominence in Swedish. In: Quaterly Progress and Status Report, vol. 41, no. 41. Department of Speech, Music and Hearing, KTH, Stockholm, Sweden, pp. 001-052.

Fant, G., Liljencrants, J., 1994. Data reduction of LF voice source parameters. In: Working Papers 43. Department of Linguistics and Phonetics, Lund University, Sweden, pp. 062-065.

Fant, G., Liljencrants, J., Lin, Q.-G., 1985. A four-parameter model of glottal flow. In: Quaterly Progress and Status Report, vol. 26, no. 4. Department of Speech, Music and Hearing, KTH, Stockholm, Sweden, pp. 001-013.

Fant, H.F., Liljencrants, J., 1981. Dynamic characteristics of voice fundamental frequency in speech and singing. acoustical analysis and physiological interpretations. In: Quaterly Progress and Status Report, vol. 22, no. 1. Department of Speech, Music and Hearing, KTH, Stockholm, Sweden, pp. 001-020.

Forney, G.D., 1973. The Viterbi algorithm. Proceedings of the IEEE 61 (3), 268-278.

Fröhlich, M., Michaelis, D., Strube, H., 2001. Sim - simultaneous inverse filtering and matching of a glottal flow model for acoustic speech signals. Journal of the Acoustical Society of America 110 (1), 479-488.

Gobl, C., 1988. Voice source dynamics in connected speech. In: Quarterly Progress and Status Report, vol. 29, no. 1. Department of Speech, Music and Hearing, KTH, Stockholm, Sweden, pp. 123-159.

Gobl, C., 2003. The voice source in speech communication. Department of Speech, Music and Hearing, KTH, Stockholm (PhD thesis).

Gobl, C., Chasaide, A.N., 1992. Acoustic characteristics of voice quality. Speech Communication 11 (4-5), 481-490.

Gudnason, J., Thomas, M.R.P., Ellis, D.P.W., Naylor, P.A., 2012. Data-driven voice source waveform analysis and synthesis. Speech Communication 54 (2), 199-211.

Hanson, D., Gerratt, B., Berke, G., 1990. Frequency, intensity, and target matching effects on photoglottographic measures of open quotient and speed quotient. Journal of Speech and Hearing Research 33, 45-50.

Hanson, H., 1995. Individual variations in glottal characteristics of female speakers. In: Proceedings of the IEEE International Conference on Acoustics, Speech, and Signal Processing (ICASSP), vol. 1, pp. 772-775.

Henrich, N., d'Alessandro, C., Doval, B., 1999. Glottal open quotient estimation using linear prediction. In: Proceedings of the International Workshop on Models and Analysis of Vocal Emissions for Biomedical Applications, pp. 12-17.

Henrich, N., d'Alessandro, C., Doval, B., 2001. Spectral correlates of voice open quotient and glottal flow asymmetry: theory, limits and experimental data. In: 7th European Conference on Speech Communication and Technology (EUROSPEECH), 2nd Interspeech Event, Aalborg, Denmark, pp. 47-50.

Henrich, N., d'Alessandro, C., Doval, B., Castellengo, M., 2004. On the use of the derivative of electroglottographic signals for characterization of nonpathological phonation. Journal of the Acoustical Society of America 115 (3), 1321-1332.

Herbst, C., 2004. Evaluation of various methods to calculate the egg contact quotient. Department of Speech, Music and Hearing, KTH, Stockholm, Sweden (diploma thesis in music acoustics).

Hill, T., Lewicki, P., 2007. Statistics: Methods and Applications. StatSoft, Tulsa, Oklahoma, USA.

Huber, S., Roebel, A., Degottex, G., 2012. Glottal source shape parameter estimation using phase minimization variants. In: 13th Annual Conference of the International Speech Communication Association (Interspeech ICSLP), 1990-9772. Portland, Oregon, USA.

Kane, J., Gobl, C., 2013a. Automating manual user strategies for precise voice source analysis. Speech Communication 55 (3), $397-414$.

Kane, J., Gobl, C., 2013b. Wavelet maxima dispersion for breathy to tense voice discrimination. IEEE Transactions on Audio, Speech, and Language Processing 21 (6), 1170-1179.

Kane, J., Yanushevskaya, I., Chasaide, A.N., Gobl, C., 2012. Exploiting time and frequency domain measures for precise voice source parameterisation. In: Proceedings of the 6th International Conference on Speech Prosody, Shanghai, China.

Karlsson, I., 1990. Voice source dynamics for female speakers. In: First International Conference on Spoken Language Processing (ICSLP), Kobe, Japan, pp. 69-72.

Keating, P.A., Esposito, C.M., 2006. Linguistic voice quality. In: Proceedings of the Eleventh Australasian International Conference on Speech Science and Technology, University of Auckland, Auckland, New Zealand, pp. 85-91.

Kominek, J., Black, A.W., 2004. The cmu arctic speech databases. In: Proceedings of the 5th ISCA Speech Synthesis Workshop, pp. 223-224.

Kreiman, J., Iseli, M., Neubauer, J., Shue, Y.-L., Gerratt, B.R., Alwan, A., 2012. The relationship between open quotient and h1(*)-h2(*). Journal of the Acoustical Society of America 124 (4), 2495.

Kreiman, J., Shue, Y.-L., Chen, G., Iseli, M., Gerratt, B.R., Neubauer, J., Alwan, A., 2012b. Variability in the relationships among voice quality, harmonic amplitudes, open quotient, and glottal area waveform shape in sustained phonation. Journal of the Acoustical Society of America 132 (4), 2625-2632.

Lanchantin, P., Rodet, X., 2010. Dynamic model selection for spectral voice conversion. In: 11th Annual Conference of the International Speech Communication Association (Interspeech ICSLP), Makuhari, Chiba, Japan.

Lanchantin, P., Rodet, X., 2011. Objective evaluation of the dynamic model selection method for spectral voice conversion. In: IEEE International Conference on Acoustics, Speech, and Signal Processing (ICASSP), Prague, Czech Republic, pp. 5132-5135.

Laver, J.D.M., 1968. Voice quality and indexical information. International Journal of Language and Communication Disorders 3 (1), $43-54$.

Laver, J.D.M., 1980. The Phonetic Description of Voice Quality, vol. 31. Cambridge University Press.

Maddieson, I., Hess, S., 1987. The effects of f0 of the linguistic use of phonation type. In: 11th International Congress of Phonetic Sciences (ICPhS), No. 67. UCLA Working Papers in Phonetics, pp. 112-118.

Maeda, S., 1982. A digital simulation method of the vocal-tract system. Speech Communication 1 (3-4), 199-229.

Marasek, K., 1997. Egg \& Voice Quality, online web resource: http://www.ims.uni-stuttgart.de/institut/arbeitsgruppen/phonetik/EGG/frmst1.htm.

Murphy, P.J., 2001. Spectral tilt as a perturbation-free measurement of noise levels in voice signals. In: 7th European Conference on Speech Communication and Technology (EUROSPEECH), 2nd Interspeech Event, Aalborg, Denmark, pp. 1495-1498. 
Obin, N., 2012. Cries and whispers - classification of vocal effort in expressive speech. In: 13th Annual Conference of the International Speech Communication Association (Interspeech ICSLP), Portland, Oregon, USA.

Ó Cinnéide, A., 2012. Phase-distortion-robust-voice-source analysis. Dublin Institute of Technology, Dublin, Ireland (PhD thesis).

Ó Cinnéide, A., Dorran, D., Gainza, M., Coyle, E., 2011. A frequency domain approach to ARX-LF voiced speech parameterization and synthesis. In: 12th Annual Conference of the International Speech Communication Association (Interspeech ICSLP), Florence, Italy, pp. 57-60.

Orlikoff, R.F., 1991. Assessment of the dynamics of vocal fold contact from the electroglottogram: data from normal male subjects. Journal of Speech, Language, and Hearing Research 34 (5), 1066-1072.

Pearson, K., 1900. On the criterion that a given system of deviations from the probable in the case of a correlated system of variables is such that can be reasonably supposed to have arisen from random sampling. Philosophical Magazine 50, 157-175.

Roebel, A., 2010. Shape-invariant speech transformation with the phase vocoder. In: 11th Annual Conference of the International Speech Communication Association (Interspeech ICSLP), Makuhari, Chiba, Japan, pp. 2146-2149.

Rothenberg, M., 1972. A new inverse-filtering technique for deriving the glottal air flow waveform during voicing. Journal of the Acoustical Society of America 53 (6), 1632-1645.

Sapienza, C.M., Stathopoulos, E.T., Dromey, C., 1998. Approximations of open quotient and speed quotient from glottal airflow and egg waveforms: effects of measurement criteria and sound pressure level. Journal of Voice 12, 31-43.

Scherer, S., Kane, J., Gobl, C., Schwenker, F., 2012. Investigating fuzzy-input fuzzy-output support vector machines for robust voice quality classification. Computer Speech and Language 27 (1), 263-287.

Strik, H., 1998. Automatic parameterization of differentiated glottal flow: comparing methods by means of synthetic flow pulses. Journal of the Acoustical Society of America 103, 2659-2669.

Strik, H., Cranen, B., Boves, L., 1993. Fitting a LF-model to inverse filter signals. In: Third European Conference on Speech Communication and Technology (Eurospeech), Berlin, Germany.

Stylianou, Y., 2001. Applying the harmonic plus noise model in concatenative speech synthesis. IEEE Transactions on Speech and Audio Processing 9 (1), 21-29.

Thomas, M.R.P., Gudnason, J., Naylor, P.A., 2009. Data-driven voice source waveform modelling. In: IEEE International Conference on Acoustics, Speech, and Signal Processing (ICASSP), Taipei, Taiwan, pp. 3965-3968.

Thomas, M.R.P., Naylor, P.A., 2009. The sigma algorithm: a glottal activity detector for electroglottographic signals. IEEE Transactions on Audio, Speech and Language Processing 17 (8), 1557-1566.

Titze, I., Talkin, D., 1979. A theoretical study of the effects of various laryngeal configurations on the acoustics of phonation. Journal of the Acoustical Society of America 66, 60-74.

Titze, I.R., 1994. Principles of Voice Production. Prentice Hall, Englewood Cliffs, NJ, pp. 354.

Vilkman, E., Lauri, E.-R., Alku, P., Sala, E., Sihvo, M., 1999. Effects of prolonged oral reading on f0, spl, subglottal pressure and amplitude characteristics of glottal flow waveforms. Journal of Voice 13 (2), 303-312.

Vincent, D., Rosec, O., Chonavel, T., 2007. A new method for speech synthesis and transformation based on an ARX-LF source-filter decomposition and hnm modeling. In: Proceedings of the IEEE International Conference on Acoustics, Speech, and Signal Processing (ICASSP), Honolulu, Hawaii, USA, pp. 525-528.

Walker, J., Murphy, P., 2007. A review of glottal waveform analysis. Progress in Nonlinear Speech Processing, 1-21.

Yeh, C., Roebel, A., 2004. A new score function for joint evaluation of multiple f0 hypothesis. In: 7th International Conference on Digital Audio Effects (DAFx), Naples, Italy, pp. 234-239.

Zivanovic, M., Roebel, A., Rodet, X., 2004 September. A new approach to spectral peak classification. In: Proceedings of the 12th European Signal Processing Conference (EUSIPCO), Vienna, Austria, pp. 1277-1280. 